\title{
$1 N-32$
}

NASA Technical Memorandum 107039

66622

A.

\section{Advanced Communications Technology Satellite (ACTS): Design and On-Orbit Performance Measurements}

F. Gargione

Lockheed Martin Astro Space

Windsor, New Jersey

R. Acosta, T. Coney, a d R. Krawczyk

Lewis Research Center

Cleveland, Ohio

September 1995

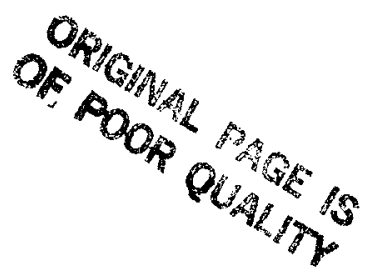

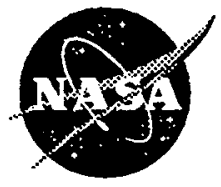

National Aeronautics and Space Administration

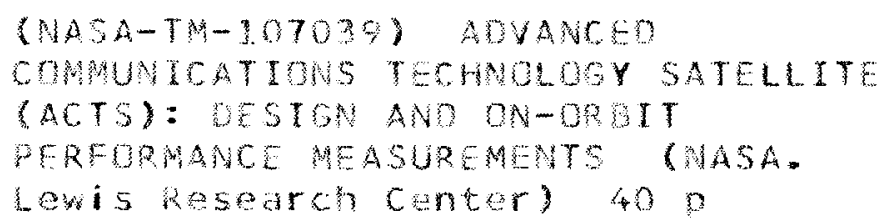




\section{ADVANCED COMMUNICATIONS TECHNOLOGY SATELLITE (ACTS): \\ Design and On-orbit Performance Measurements \\ F. Gargione; Lockheed Martin Astro Space}

R. Acosta, T. Coney and R. Krawczyk; NASA Lewis Research Center

\section{ABSTRACT}

The Advanced Communications Technology Satellite (ACTS), developed and built by Lockheed Martin Astro Space for the NASA Lewis Research Center, was launched in September 1993 on the Shuttle STS 51 mission. ACTS is a digital experimental communications test bed that incorporates gigahertz bandwidth transponders operating at $\mathrm{Ka}$ band, hopping spot beams, on-board storage and switching, and dynamic rain fade compensation. This paper describes the ACTS enabling technologies, the design of the communications payload, the constraints imposed on the spacecraft bus, and the measurements conducted to verify the performance of the system in orbit.

\section{INTRODUCTION}

The ACTS satellite was designed to provide Demand Assigned Multiple Access (DAMA) digital communications with a minimum switchable circuit bandwidth of $64 \mathrm{Kbps}$, and a maximum channel bandwidth of $900 \mathrm{MHz}$. It can, therefore, provide service to thin routes as well as connect fiber backbones in supercomputer networks, across oceans, or to restore full communications in the event of national or manmade disasters.

Although designed for fixed service digital communications, the high gain afforded by the ACTS spot beams and the ability to provide a bent pipe transponder (with the switch matrix held in a fixed state) has also permitted a large number of terrestrial and airborne mobile experiments.

ACTS has proven to be a true orbiting multiservice communications laboratory affording not only a multitude of advances in digital communications, but also the gathering of propagation statistics necessary for the implementation of reliable, $\mathrm{Ka}$ band operational systems.
The ACTS System

The ACTS system, shown in Figure 1, is composed of the ACTS satellite, now in geostationary orbit at $100^{\circ}$ West longitude; the Satellite Control Center located at Lockheed Martin Astro Space in East Windsor, New Jersey, which controls the spacecraft and provides 24 hour monitoring of health status; the Telemetry Tracking and Command Station located at the NASA Lewis Research Center in Cleveland, Ohio, which uplinks commands and receives telemetry at $\mathrm{Ka}$ band. The NASA Master Control Station (MCS), which controls the $\mathrm{Ka}$ band communication network and directs all the experiments, is also located at the NASA Lewis Research Center.

User terminals located throughout the United States complete the ACTS system. These terminals were developed by NASA and are available to users either on loan from NASA or by direct purchase.

The available terminals fall into three broad categories, depending on whether they are used with the Baseband Processor (BBP), the Switch Matrix, or for propagation measurements, features of the satellite that will be described later in the paper.

For the BBP mode of operation, the NASA Ground Station, developed and built by COMSAT, provides control of the TDMA network and directs the traffic to/from eighteen T1 VSAT terminals, developed by Harris, and deployed all over the USA. The NASA Ground Station also serves as a traffic terminal. The U.S. Army has purchased seven $\mathrm{T} 1$ terminals, some of which have been ruggedized so they can be transported to simulate battlefield conditions where field deployment and network acquisition are very critical.

For the Microwave Switch mode of operation, a number of terminals have been developed as described below: 
a) A Land Mobile Terminal developed by JPL for NASA and used to conduct experiments first in the Southern California area and later in other areas of the country. The data rate supported ranges from 2.4 to $64 \mathrm{Kbps}$.

b) Ultra Small Aperture Terminals developed by NASA Lewis and Southern California Edison for Supervisory Control and Data Acquisition (SCADA) purposes. They have a data rate of about $2 \mathrm{Kbps}$ and will be used for monitoring the status of power lines.

c) An Aero-Mobile Terminal developed by NASA Lewis and JPL to provide voice and data to aircraft. It was mounted on a Lewis airplane to conduct the experiment and flown to various U.S. cities.

d) A Broadband Aero-Mobile Terminal is being developed by JPL with Rockwell to provide broadband video, voice and data to aircraft.

e) A Link Evaluation Terminal (LET) developed by NASA Lewis to evaluate the ACTS transponders. It has a data rate of 220 Mbps. In addition to characterizing the transponders, it has been used to perform measurements to locate the center of the Cleveland spot beams.

f) The High Data Rate Terminal (HDR) being developed by NASA in conjunction with ARPA to provide 622 Mbps links between supercomputers for parallel processing experiments. It will also be used for HDTV experiments by PBS.

For propagation measurements, NASA has developed a number of receive-only terminals to be used to characterize the $\mathrm{Ka}$ band signal attenuation due to rain. Eight terminals have been built and are deployed in significant rain zone areas to collect statistical data on the frequency and severity of rain fade phenomena.

\section{THE ACTS TECHNOLOGIES}

The ACTS satellite communications system contains new technologies that will become the standard of communications satellites of the future.
They are:

1) hopping spot beams

2) onboard switching

3) $\mathrm{Ka}$ band transmission

4) wide bandwidth channels

5) rain fade compensation

6) $\mathrm{Ka}$ band propagation beacons

The advantages that these technologies bring to the field of satellite communications are discussed in the following sections of this paper.

\section{Hopping Spot Beams}

Forerunners to the ACTS satellite have used broad coverage beams that provided relatively low signal levels, requiring large ground receiving antennas and even larger uplink antennas, to the point that the uplink could only be done from very specialized and expensive stations. Spot beams, by contrast, concentrate the energy into small beams with very small footprints, providing in the case of ACTS an improvement of $20 \mathrm{~dB}$ in signal level, resulting in small diameter antennas and higher throughput for each earth station. The use of spot beams, however, necessitates a large number of stationary beams or a rather small number of hopping beam families to cover a large geographic area.

The beam locations were chosen on the basis of population density, climate diversity, and availability of data sources commensurate with the high bandwidth channels in the ACTS satellite.

The beams are broken into fixed beams for use with the Switch Matrix, and two families of hopping spot beams for use with the Baseband Processor. However, either type of beam can work with either type of switch to provide on-demand operation in response to traffic needs.

The two families of hopping beams cover two contiguous sectors with uniform gain, a number of isolated spots, and finally a steerable beam to cover Hawaii, Alaska and the entire hemisphere. The fixed and hopping beams all have a $0.3^{\circ}$ beamwidth, while the steerable beam has a $1^{\circ}$ beamwidth due to the smaller size of the steerable reflector. The ACTS spot beam coverage is shown in Figure 2. 
Onboard Switching

The ACTS satellite has two types of onboard switching; a Switch Matrix to connect the three fixed beams or the two families of hopping beams and a Baseband Processor with storage to control traffic between the two families of hopping beams.

The Switch Matrix provides bent pipe or dynamic switching through transponders having a bandwidth of $900 \mathrm{MHz}$. When the Switch Matrix is used dynamically, it has a frame of one millisecond with switching states of one microsecond granularity. A frame of 32 milliseconds is also available for low data rate terminals or for applications requiring longer dwell time for each configuration.

In the case of the Baseband Processor, each hopping beam family operates at a maximum throughput of $110 \mathrm{Mbps}$, with a frame of 1728 slots, for a total TDMA throughput of $220 \mathrm{Mbps}$. One inherent advantage of baseband processing is that only those beams that are active need to be visited, thus providing demand assigned multiple access (DAMA) channels that make maximum use of available resources on the satellite.

\section{$\underline{\mathrm{Ka} \text { band Transmission }}$}

The demand for wireless communications, either terrestrial or by satellite, has created a shortage of spectrum which will become even more acute as less developed areas of the world increase the demand for communications. To alleviate this crowding of the spectrum, ACTS was designed to use $\mathrm{Ka}$ band frequencies ( $30 \mathrm{GHz}$ uplink and 20 $\mathrm{GHz}$ downlink), thus developing the technology and the components necessary to build reliable transponders on the satellite and transmitting and receiving stations on the ground.

$\mathrm{Ka}$ band has the advantages of increasing the number of satellites that can be placed on the equatorial arc because of the narrow beamwidth that results from the use of the higher frequency, and of reducing the size of the satellite and ground antennas required for any given gain.

One disadvantage of $\mathrm{Ka}$ band, however, is its susceptibility to signal loss due to rain, which dictates that suitable measures be taken to compensate for the loss of signal in order to provide reliable, high availability communication channels.

\section{Wide Bandwidth Channels}

The use of $\mathrm{Ka}$ band allows the implementation of very high bandwidth channels. In the case of ACTS, the transponders have a bandwidth of $900 \mathrm{MHz}$. The availability of such wide channels makes possible the interconnections of supercomputers, the high speed distribution of high density imagery for applications such as remote medical diagnosis, weather and earth resource studies and topographic applications. This large bandwidth, available on demand, should secure for satellites a pre-eminent place in the implementation of the National Information Infrastructure.

\section{Rain Fade Compensation}

In order to provide reliable communications, compensation must be provided for the loss of strength, or fading, of the $\mathrm{Ka}$ band signal due to rain. Two techniques are used on the ACTS satellite: Increased output of the transmitters and the use of data rate reduction along with convolutional coding.

Increased transmitter power is used in conjunction with the Switch Matrix mode of operation. ACTS has enough RF power to provide a fixed $8 \mathrm{~dB}$ link margin on the downlink. The uplink margin is up to $18 \mathrm{~dB}$ and can be applied on demand by modulating the power of the ground transmitter.

For the Baseband Processor mode of operation, fading on the downlink is compensated by supplying $3 \mathrm{~dB}$ of clear weather margin, supplemented by $4 \mathrm{~dB}$ from the use of convolutional coding and $6 \mathrm{~dB}$ obtained from frequency and data rate reduction. For the uplink, the clear weather margin is $5 \mathrm{~dB}$, so a total of $15 \mathrm{~dB}$ can be provided when coding and data rate reduction are invoked. The fade compensation for both uplink and downlink is applied on demand only to those stations that require it.

The Baseband Processor provides fade compensation by setting aside a number of slots in a "fade pool". When a station experiences fading, its burst is moved from the clear part of the frame to the fade pool area where a fourfold increase in the number of slots can be provided without disturbing the 
other stations burst time plans. The size of the "fade pool" is a system parameter that is adjusted as needed to provide adequate service and still maintain good frame efficiency.

\section{Propagation Beacons}

The ACTS satellite is equipped with stable beacons operating at 20 and $27 \mathrm{GHz}$ and covering primarily the Continental United States.

The beacons are monitored by a number of propagation-only stations to provide the data for developing a comprehensive rain fade model that can be used to optimize the type and amount of fading compensation that would be needed for a given class of service in future operational systems.

\section{THE ACTS SPACECRAFT}

The ACTS spacecraft, shown in Figure 3, is composed of three main elements: The Bus, the Multibeam Antenna (MBA) and the Communications Payload.

\section{The Bus}

The Bus is a Series 4000 three-axis stabilized Lockheed Martin standard bus, whose tighter pointing accuracy of $0.025^{\circ}$ in pitch and roll is achieved by autotracking on the command carrier from Cleveland.

The yaw pointing accuracy of $0.15^{\circ}$ is achieved by using sun sensors on the east and west faces, and by an on-board Yaw Estimator Program that maintains pointing when the sun sensors are not illuminated by the sun.

Table I shows the key specifications of the bus. The specifications for the MBA and the Communications Payload as shown in Table II.

\section{The ACTS Communications Payload}

A detailed block diagram of the ACTS Communications Payload is shown in Figure 4. Uplink signals can be received through three fixed spot beams, located at Tampa, Cleveland and Atlanta, which in conjunction with the Microwave Switch Matrix form the High Burst Rate (HBR) system, capable of serving three demand-assigned TDMA channels with bandwidth of $900 \mathrm{MHz}$ each.
Uplink signals can also be received through two families of hopping spot beams steered through East and West Beam Forming Networks which, in conjunction with the Baseband Processor, form the Low Burst Rate (LBR) system, intended to provide demand assigned TDMA thin route circuits of $64 \mathrm{Kbps}$, which can be aggregated to provide wide bandwidth channels up to the maximum capacity of $110 \mathrm{Mbps}$ for each of the two channels of the Baseband Processor.

The Cleveland uplink fixed beam is equipped with a special tri-mode hom that also receives the command carrier, which is processed through an Autotrack Modulator and Receiver to provide an error signal to the spacecraft that maintains the receive antenna locked on Cleveland, thus providing the fine pointing of $0.025^{\circ}$ needed for the small diameter $(0.30)$ spot beams.

Each of the uplinked signals can be directed to any of four receivers by means of the Waveguide Input Redundancy Switch (WIRS). The receivers use HEMT inputs and downconvert the input from $30 \mathrm{GHz}$ to the 3 $\mathrm{GHz}$ IF used by the Microwave Switch Matrix (MSM) or the BBP. The receivers provide saturated outputs to the MSM, and linear outputs to the BBP Demodulator. The IF outputs of the receivers can be directed to either the MSM or the BBP by means of a Receive Coaxial Switch Assembly. After processing by either the MSM or the BBP, signals are switched through the Transmit Coaxial Switch Assembly to any of four upconverters which upconvert the IF signals to $20 \mathrm{GHz}$ for amplification in the 46 Watt Traveling Wave Tube Amplifiers (TWTA). The outputs of the TWTA can be directed by the Waveguide Output Redundancy Switch to any of the fixed beam or scanning beam ports of the Transmit Antenna.

The key specification parameters of the payload are shown in Table II, but some additional information on the MSM and the $\mathrm{BBP}$ will be provided here

The MSM is a four-by-four matrix providing crosspoint switching from any input to any, or all, outputs. Its bandwidth is $900 \mathrm{MHz}$, and the switching speed is 100 nanoseconds. It provides a $1 \mathrm{msec}$ or $32 \mathrm{msec}$ TDMA frame with switching at the $1 \mu \mathrm{sec}$ or $32 \mu \mathrm{sec}$ boundaries. Switching is controlled by a ping-pong memory, so that while the 
foreground memory controls the present TDMA plan, the background memory can be programmed through the command link to implement the next TDMA plan, which is invoked by swapping the control from the foreground to the background memory. The memory provides telemetry of the active switch states for ground verification before swapping of control.

The Baseband Processor is much more complex than the MSM. It demands an exact protocol, specific signal modulation (SMSK) and frequency, and burst timing within a 60 nanosecond window. It provides demodulation, convolutional decoding, storage, switching, convolutional coding and remodulation of thin route circuits with 64 Kbps bandwidth. Convolutional coding and decoding is coupled with data rate reduction to achieve on-demand margin improvement of $10 \mathrm{~dB}$ to selected uplink and downlink stations experiencing fading due to rain. The $\mathrm{BBP}$ is a memory-mapped processor receiving its operating instructions via in-band commands (orderwires) from the Master Control Station located in Cleveland. The $\mathrm{BBP}$ has a $1 \mathrm{msec}$ frame and can handle a total thruput of $220 \mathrm{Mbps}$ with its two channels.

Both the MSM and the BBP control the switching of the hopping beams as dictated by the TDMA frame, dwelling only at those locations requiring service for a true demandassigned operation. The hopping beams thus switch very fast, typically in less than $1 \mu \mathrm{sec}$, and can visit a maximum of 40 dwells in a given $1 \mathrm{msec}$ frame.

Although a part of the Command/Ranging and Telemetry Subsystem, two very stable beacons operate in conjunction with the payload to provide fade data. The beacon at the uplink frequency is actually set at 27.505 $\mathrm{GHz}$ to avoid interference with the communications signals. The downlink frequency beacon, set at $20.185 \mathrm{GHz}$, also serves as a carrier for telemetry and ranging. Telemetry data modulates a $64 \mathrm{kHz}$ subcarrier which is always present. An unmodulated $32 \mathrm{kHz}$ carrier is used as a placeholder when not ranging, and is replaced by ranging tones when ranging. The amplitude of the ranging tones is adjusted to produce the same carrier modulation as the $32 \mathrm{kHz}$ subcarrier, to maintain the beacon carrier amplitude constant.

\section{The Multibeam Antenna}

The ACTS MBA consists of two offset Cassegrain antennas (one transmit and one receive) and an antenna support assembly. The two antennas are nearly identical in an RF sense, with the larger $20 \mathrm{GHz}$ transmit main reflector having a diameter of $3.3 \mathrm{~m}$ and the smaller $30 \mathrm{GHz}$ receive reflector a diameter of $2.2 \mathrm{~m}$. Aside from a common antenna support assembly, each antenna consists of three major sections: A main reflector, an assembly consisting of one front and one back subreflector, and a pair of feed assemblies; one for horizontal, and the other for vertical polarization.

Because of its strength, light weight, and low coefficient of thermal expansion characteristics, graphite epoxy finds wide use in the MBA. The entire MBA weighs 932 lbs. The antenna support assembly, fabricated from graphite epoxy tubes with titanium fittings, makes up about half its weight. The basic geometry of the two antennas is shown in Figure 5.

ACTS MBA beam topology (uplink and downlink) can be divided into a set of four independent beam families; the East receive (uplink), East transmit (downlink), West receive (uplink) and West transmit (downlink). The ACTS MBA beam coverage is shown in Figure 2, which also shows an example of the larger-area but lower-gain coverage of the separate steerable-beam antenna.

The ACTS MBA incorporates a total of two main reflectors and four subreflectors. High surface accuracy of these reflectors is critical to the RF performance of the MBA. The largest of these units is the $3.3 \mathrm{~m}, 20 \mathrm{GHz}$ transmit main reflector. This was formed on a precision mold and consists of six-ply graphite epoxy front and back facesheets with a Kevlar honeycomb core. The reflector shell is supported by a backing rib structure of similar construction. This reflector weighs about $80^{\circ} \mathrm{lbs}$. and has an rms accuracy of 3 mils. The $30 \mathrm{GHz}$ receive main reflector is similar in construction, weighs about $30 \mathrm{lbs}$. and has the same surface accuracy. The transmit and receive back subreflectors are also similar in construction but are hyperbolic rather than parabolic. 
The construction of the front subreflector is very different. Because the front subreflector is required to transmit $R F$ at one polarization and to reflect the orthogonal polarization, it is necessary to make the basic reflector as transparent to RF and also mechanically rigid. The front subreflector shells are formed from Astroquartz facesheets and a Nomex core. Each shell has a thickness of approximately three-quarters of a wave-length at the frequency in consideration. There is no ribbing behind the central section of the subreflector, but a peripheral rib provides rigidity. Each front subreflector has formed copper gridding on its front facesheets to serve as a polarization-selective reflector. The two-way insertion loss of each front subreflector is below $2 \mathrm{~dB}$. Reflection loss is about $0.25 \mathrm{~dB}$.

The feed assemblies incorporate three different types of feed horn. These are illustrated in Figure 6 . Figure 7, shows a multiflare horn designed to provide a circularly symmetrical primary pattern needed to provide efficient illumination of the reflector. This horn is used for the three trunking beams and provides an edge taper of $-17 \mathrm{~dB}$ at $+/-13^{\circ}$, resulting in very low sidelobe levels (more than $30 \mathrm{~dB}$ below peak at two half-power beamwidths from peak). A multi-flare horn was preferred to either a dual mode horn or a corrugated horn, because of its narrow bandwidth, low weight and ease of manufacturing. Figure 8 , shows the multiflare horn designated for the scan spot beams It is similar to the trunking horn, except for an edge taper of $-10 \mathrm{~dB}$ at $+/-13^{\circ}$. The scan array of horns are simple single-flare horns with an edge taper of about $3 \mathrm{~dB}$.

\section{Autotrack System}

The autotrack system processes the azimuth (pitch) and elevation (roll) pointing error signals which are produced in the tracking feed system on the receive antenna. These signals are induced as higher order modes in the feed by angular offsets of the propagation axis of the incoming radiation from the axis of the Cleveland receive horn. Both error signals are time multiplexed on to the Cleveland command uplink signal by the autotrack modulator and coupler. The combined signals then pass through the low Noise Receiver Downconverter (NRD) and Intermediate Frequency Module (IFM) where they are downconverted and amplified. In the
IFM, the autotrack signal is separated from the communication signal by filtering and sent to the autotrack receiver. Analog azimuth and elevation error signals are demultiplexed in the Autotrack Receiver (ATR), and sent to the Analog to Digital Converter which resides in the Intermediate Frequency Switch Matrix (IFSM). These signals are then sent to the attitude system processor (ASP) which uses this information in pointing the spacecraft and to the ground system which can monitor the data and assess tracking system performance.

\section{THE ACTS TEST PROGRAM}

\section{Ground Testing}

The ACTS satellite was subjected to a development and test flow designed to retire risks at the earliest opportunity to insure the success of the satellite in orbit.

To verify the design of the new technology, engineering models of all boxes were built, tested and integrated into an Engineering Model Payload, a single channel transponder with a non-BBP and a reduced size switch matrix, used for the purpose of characterizing the communication channel, debugging the test software for the payload, and as a STE for the debug of the Master Control Station TDMA software.

For the spacecraft build, all parts were procured or screened to S-level specifications; all boxes were subjected to qualification/acceptance level testing; all subsystems were integrated and tested individually and as part of the overall satellite; the advanced communications payload including the BBP was subjected to thermal vacuum cycling and performance tests individually and again as part of the satellite; the entire satellite was subjected to thermal. EMI, modal, spin balance, vibro-acustic, appendage deployments, and the thermal-vacuum tests to verify its electrical performance and mechanical integrity.

At the completion of this cycle of testing, a comprehensive system test was conducted by assembling the ground equipment and operating the spacecraft to insure compatibility with all the control equipment, such as the Master Control Station and the TT\&C station, and the communications equipment, such as the VSAT TDMA stations operating with the $\mathrm{BBP}$ or the Link Evaluation Terminal 
operating with the Switch Matrix. Also, after system test, a special switching test was performed to verify that the switching time of each hopping spot beam met specifications.

The ACTS System Test proved especially valuable because it revealed a ground software problem that had not been uncovered during previous testing with the Engineering Model of the payload.

The system test also uncovered some command dropouts in the high rate command link, used normally to control the payload configuration and also to reprogram the BBP memory to a backup configuration. This backup is only used to work around a double failure of the normal BBP input.

Both of these discrepancies were resolved and retested successfully before the spacecraft was shipped to the launch site. Also, both discrepancies would have been very difficult to diagnose after the spacecraft had reached orbit, demonstrating the value of the test program in retiring risks and insuring the delivery to orbit of a very sound satellite that has so far exceeded its performance requirements.

\section{$\underline{\text { On-Orbit Bus Test Results }}$}

The ACTS Spacecraft Bus borrowed very heavily from the Lockheed Martin Astro Space commercial communication satellite heritage in order to maximize the available resources for the development of the new communication technology. Even so, some areas of the bus needed to be improved in order to provide a stable platform with the fine pointing capability necessary to support spot beams with $0.3^{\circ}$ beamwidths.

In fact, the pointing accuracies $\left(0.025^{\circ}\right.$ in pitch and roll, and $0.15^{\circ}$ in yaw) required of the ACTS platform are at least a factor of four tighter than used in conventional CONUScoverage geosynchronous satellites. Since the tighter pointing requirements for pitch and roll could not be met using a conventional Earth Sensor Assembly (ESA), ACTS employs a RF pointing system (Autotrack) utilizing the $\mathrm{Ka}$ band command carrier from the Master Control Station in Cleveland.

The improvement in pointing accuracy in pitch and roll using RF pointing is shown in Figures 9 and 10 respectively, where the RF pointing is compared with the ESA. The disturbance in the RF pointing occurring at 1420 GMT, which causes the pitch and roll angles to oscillate, is due to thermal distortion of the Astroquartz front subreflector surface, caused by the heating of the sun as it travels across the surface. The time of occurrence and amplitude of the disturbance varies seasonally as the sun changes position with respect to the spacecraft. For yaw, the ACTS spacecraft employs two sun sensors on the East and West faces of the spacecraft to provide a sun-pointing reference during the sun viewing period and a software estimator that provides the control signal during the time that the sun sensors are not viewing the sun. Figure 11 shows the yaw angle.

The thermal subsystem design was also very demanding, necessitating the use of heat pipes to remove heat from the TWTA and BBP and supplementary heaters to warm up the payload panels before resuming operation after eclipse, when the payload is off (by design) in order to limit the weight allocated to the batteries.

In this area, extensive modeling and simulation of all critical components within the spacecraft was performed to be sure that the components temperatures could be maintained within the acceptance limits. The modeling predicts were then compared to actual measurements in vacuum where the spacecraft was operated in conditions that simulated the worst cast heating and cooling that the various spacecraft surfaces would see during each season. The measurements made during this test were compared with the predicts and found to be within $\pm 5^{\circ} \mathrm{C}$. In orbit results have confirmed both the predicts and test results to be within less than $\pm 5^{\circ} \mathrm{C}$.

\section{Payload On-Orbit Test Results}

The performance assessment of the $\mathrm{Ka}$ band payload started with individual turn-on and monitoring of each box or subsystem. For many units this was accomplished by an automated test, adapted from ground test software, that executed the appropriate commands and verified proper telemetry response. The initial on-orbit tests were intended to verify the basic health and functionality of the primary units and subsystems to allow the more comprehensive system level tests to get underway with confidence. The following paragraphs 
describe the initial testing plus some significant results gathered since then.

The ACTS includes redundant $\mathrm{Ka}$ band beacon transmitters to provide the normal spacecraft telemetry and ranging functions while producing a stable downlink signal to allow propagation measurements. This beacon is a $20.185 \mathrm{GHz}$ transmitter that is modulated with telemetry and either a placeholder subcarrier or a ranging signal with the appropriate modulation index to maintain near constant carrier power. Spectrum analyzer plots taken at the NGS indicate that the carrier level varies by $0.5 \mathrm{~dB}$ or less for these two operational modes. ACTS also includes redundant $27.505 \mathrm{GHz}$ unmodulated beacons, designated as UFB 1 and UFB 2, to allow propagation measurements in the uplink communications band. The expected received signal levels for both the telemetry and uplink beacons were derived from ground test data for the beacons' RF output and measured gains for the CONUS coverage antenna. UFB 1 was noted to be $4 \mathrm{~dB}$ lower than expected when initially turned on. After additional measurements and intensive investigation which yielded no conclusive reason for the reduced level, UFB 2 was tuned on in place of UFB 1. As indicated in Table 1, the signal level for the telemetry beacon and UFB 2 were in good agreement with the expected levels. To date, the beacon frequency drift has been better than $-7400 \mathrm{~Hz}$ per year, well within specifications. The telemetry margin is at least $14 \mathrm{~dB}$, as demonstrated by an acceptable few minutes outage due to occasional heavy rain at Cleveland.

The turn-on test provided only a basic health check of the BBP, but did provide confidence to proceed with subsequent system level tests that thoroughly exercised the BBP. This extremely complex subsystem has accumulated well over 15,000 hours operation and 160 on/off power cycles due to limited battery power during eclipses.

The payload master oscillator/frequency generator was activated on September 24 , 1993, to provide a stable clock and local oscillator source that is distributed to the payload units. This clock/oscillator has remained on continuously, accumulating 16,000 hours to date using battery power during the eclipse season. Although we cannot monitor this units' stability directly, system operation has demonstrated many months between clock corrections.

The autotrack receiver subsystem processes the continuously maintained command carrier signal to provide the error signals for precise spacecraft pointing in pitch and roll axis. The initial health check of the autotrack receiver monitored its automatic gain control level during incremental reductions in uplink signal level to verify proper response. Observations since that time have demonstrated valid autotrack error signals with $22 \mathrm{~dB}$ fades during severe rain events.

The payload uplink paths include the receive antenna elements, East and West Beam Forming Networks (BFN) and Low Noise Receivers (LNR) to implement four receive channels. The payload downlink paths include four transmit channels of Upconverters and TWTAs, East and West BFNs and the transmitting antenna elements. Initial testing of payload uplink and downlink paths took advantage of the ACTS T1-VSAT at Lockheed Martin Astro Space, within the scan area of the East BFN. The NGS at NASA Lewis in Cleveland, $\mathrm{OH}$, is within the scan area of the West BFN. The Link Evaluation Terminal (LET), also at NASA Lewis, communicates via the Cleveland fixed beam. Figure 12, shows the ground stations used to support the initial payload on-orbit tests.

The LET provides the necessary uplink test signals and downlink measuring equipment to checkout and characterize the MSM path through the payload. To accomplish these tests the MSM was programmed by ground command to establish the required uplink to downlink connections within its IF crossbar architecture.

The downlink path checkout used a test signal uplinked from the LET which was routed to each TWTA. This also verified the receive path from the Cleveland fixed horn through LNR 3. Monitoring the received signal level at the three stations verified functionality of the payload downlink paths through four TWTAs and the selected scan spots of the East West transmit BFNs. The expected signal level received by each site was calculated based on spacecraft ground test data for TWTA output power and Multibeam Antenna range test data for antenna gain. 
Table III, shows expected and measured values in good agreement. TWTA output levels have remained steady at approximately 46 watts providing a peak EIRP of 58 to 69 $\mathrm{dBW}$ to the various downlink beams. TWTA parameters, as monitored via telemetry, have remained stable with over 15,000 hours on each of the three normally used channels and there have been no spurious shutdowns.

An uplink path calibration from the LET was run on receive channel 3 using a $C W$ level detector that can be connected at the IF output of the LNR. The measured levels were $2 \mathrm{~dB}$ greater than predicted by the nominal link budgets. It should be mentioned that this path was also used to characterize Multibeam antenna receive patterns. The $\mathrm{CW}$ tone, uplinked from the LET, was also swept across the communications band. This initial characterization of transponder frequency response showed good agreement with ground testing across the $900 \mathrm{MHz}$ bandwidth. The LET also transmitted modulated (bursted) data at $110 \mathrm{Mbps}$ and $220 \mathrm{Mbps}$ while achieving a BER of 1x10-7. Subsequent system level tests, using recently developed HDR terminals, have demonstrated excellent data quality at $622 \mathrm{Mbps}$.

The uplink path functional testing was then run on channels 2 and 4 . A test signal from the NGS through the West BFN and LNR2 was looped back to the NGS via downlink channel 2 to verify the integrity of uplink channel 2. Similarly, a test signal from the T1-VSAT through the East BFN and LNR4, was routed back to Cleveland to verify uplink channel 4. Uplink channel 1 was demonstrated to be functional several months after launch, when an experimenter in Atlanta came on-line.

The remaining MSM switches not utilized for the uplink/downlink tests were functionally verified by monitoring the response of signal level detectors at the MSM outputs to the appropriate switch closure commands and the resultant change in noise level. Subsequent system level tests have utilized connectivity through 11 of the 16 switches, to date. The MSM control memories have also been programmed for TDMA connectivity with 1 and $32 \mathrm{msec}$ frame durations to support high data rate experiments.

The integrity of the control interface to the BFNs was also verified during the uplink/downlink tests by selecting the proper scan beam for Cleveland and East Windsor. This demonstrated static control of the BFNs while subsequent system level testing demonstrated sub- $\mu$ sec dynamic control by hopping the beams among several T1-VSATs in a network. Thus far, about half of the 48 BFN scan locations have been utilized for experiment support.

The Steerable Beam Antenna (SBA) is intended to support those locations not covered by the fixed or scanning beams. The SBA was tested using a CW uplink from the LET with the NGS monitoring the loopback signal. The SBA was commanded from its initial launch position and pointed to provide a peak signal at Cleveland. Table III, also shows nominal performance for this downlink path. The SBA was then commanded to trace out a rectangular path in each quadrant starting from Cleveland to verify pointing control and position telemetry. The SBA pointing has proven to be very repeatable during network level testing, by correlating the positioning indicated by shaft potentiometer telemetry with T1-VSAT bit error rates. The SBA has supported experiments in Alaska, Hawaii and South America. Recently, ground software was developed to allow the SBA to perform closed loop tracking of an aircraft in flight based on GPS inputs. To date, the NASA Kuiper Observatory, flying out of NASA Ames, has been successfully tracked using this new software.

\section{MBA On-Orbit Measurements}

During the various MBA test procedures, the space-craft may make use of either of two different orientation-sensing systems. These are:

1) Autotrack System; The spacecraft rotates in pitch and roll in increments of $0.01^{\circ}$ with a dynamic range of $+/-0.12^{\circ}$

2) Earth Sensor System; The spacecraft rotates in pitch and roll increments of $0.02^{\circ}$ with a dynamic range of at least $+/-$ $1.0^{\circ}$

Both systems directly control the momentum wheel assemblies (East-West/pitch) and magnetic torquers (North-South/roll) of the spacecraft attitude control system, providing 
spacecraft attitude control system, providing the capability of rotating the spacecraft in the desired directions.

\section{Sources of Error}

The on-orbit MBA tests include similar types of error sources as more conventional nearfield measurements. These are the uncertainties due to ground station performance (such as gain, transmission power, receiver instabilities and polarization purity), misalignments (such as satellite and ground station pointing error) and uncertainties in satellite RF systems (such as receiver noise figure, TWTA outputpower level, etc.).

Propagation effects are compensated based on measurements of received beacon power. The satellite movement, with respect to Earth as a function of time, is also a source of error. Although this movement is small (less $0.1^{\circ}$ ), it can still have significant effect on the measurements.

\section{Thermal Distortions}

The types of on-orbit thermal distortion effects on MBA pointing can be classified as:

\section{1) Rapid Variation \\ 2) Diurnal Variation \\ 3) Quasistatic Variation}

The rapid variation thermal distortion is caused by a nonuniform sun flux illumination of the front subreflectors (transmit and receive). This thermal condition causes large temperature gradients in the subreflectors. These temperature gradients cause local distortions of the subreflectors surface of the order of 0.060 " (peak). This produces beam movement or wandering of approximately $0.1^{\circ}$ as indicated by autotrack system.

The diurnal variation thermal distortion is observed when the reflector rotates (pitch) West, starting at $0200 \mathrm{Z}$; reaching their maximum Westward movement of approximately $0.2^{\circ}$ at $0800 \mathrm{Z}$ and returning to the starting point by $1400 \mathrm{Z}$. The diurnal variation is independent of whether the $S / C$ is controlled by the autotrack or ESA system.

The quasistatic variation is evident by $a+1-$ $0.04^{\circ}$ variation in pitch and $+1-0.02^{\circ}$ variation in roll over an approximate two week period as measured by the autotrack system when
ESA is in control of the $S / C$. The resultant "drift" is caused by the coarse point stability and by the thermally induced effects on the ESA system.

Rapidly Varying Thermal Effects on the East Receive Pointing Stability

The East receive beams are represented by the autotrack (uplink-East) pitch and roll error signals. The autotrack signatures (pitch and roll) as a function of the time of year are presented in Figure 13. The data was taken approximately every 30 days. The corresponding subreflector temperature profiles are shown in Figure 14. The data clearly establishes correlation between the East receive $(30 \mathrm{GHz})$ beam pointing error and font subreflector temperature gradients. The period of large subreflector temperature gradients occurs between $1300 \mathrm{Z}$ and $2200 \mathrm{Z}$. This period of time is defined as the rapidly varying thermal period (RVTP). One year comparison between the East beams pointing (autotrack pitch and roll) error signal, and its corresponding subreflector temperature profile are presented in Figures 15 and 16, respectively. This data indicates that the rapidly varying thermal distortions are essentially periodic (365 day period). This can be explained by analyzing the subreflector orientation and shadowing relative to the sun as function of time of the year (See Figure 17).

Rapidly Varving Thermal Effects on the East Transmit Pointing Stability

The measurements for the East transmit (downlink) beam pointing error are based on ground RF (Eb/No - energy per bit per noise density) received signals. A typical East transmit (downlink) RF signal recorded by the NASA LET is shown in Figure 18. The corresponding subreflector (on-orbit) temperatures as a function of time of year are depicted in Figure 14. The data shows correlation between the reflector temperature gradients and receive downlink signal degradation during the rapidly varying time period. It can be observed that the receive and transmit rapidly varying events are separated by 2 hours. Other East downlink beam signals recorded by the other T1- VSATs also show correlation between subreflector temperature profile and received $R F(E b / N o)$ signal (See Figure 19). 
Rapidly Varying Thermal Effects on the West Transmit and Receive Pointing Stability

A typical West downlink beam recorded by the NGS as a function of time of the day is shown in Figure 20. This RF signature shows no fast varying signal response during the rapidly varying time period. The other West downlink beam recorded by T1-VSATs are shown $n$ Figure 21. The recorded data on transmit and receive East beams show no correlation between subreflector temperature gradients and signal degradation during the rapidly varying events.

Diumal Variation Thermal Effects on the East and West Transmit Pointing Stability

The effects of the diurnal variation on MBA pointing can be represented by the data recorded on the East and West transmit RF signal received by the NGS and T1-VSATs (See Figures 20 and 21). The diurnal variation affects the West and East downlink beams equally. The period for the Diumal variation occurs between $0000 \mathrm{Z}-1300 \mathrm{Z}$. This period of time is defined as the diumal variation thermal period (DVTP). No correlation was observed between the temperature on the main reflector surface or spacecraft body and RF signal variation. It is believed that the diurnal variation is caused by a rotation in pitch of the transmit main reflector due to thermal effects on the antenna assembly. No receive East or West diurnal variation has been observed or recorded at any time.

Quasistatic Effect on the MBA Pointing Stability

The quasistatic effect on all MBA beams (uplink and downlink) is caused by the coarse control (stability, accuracy, etc.) and by thermally induced effects on the ESA. All MBA uplink and downlink beams will wander by the same amount due to the quasistatic effect. The MBA pointing error can be represented (S/C in ESA control) by the autotrack pitch and roll error angles at a given time of day (e.g., 0800Z and 1600Z). The resulting functional representation of the MBA pointing error as function of time of the year is presented in Figure 22. The data was recorded (pitch and roll error signals) every 2 days for 365 days.
Most MBA experiments show correlation between the transmit and receive antennas when the ACTS S/C is controlled by the ESA. The data shows the MBA pointing error is worse at the equinox and minimum at solstice. It is difficult to independently evaluate the effects of MBA pointing error due to thermal distortions (other that rapidly varying and diurnal), ESA thermal and yaw errors. These effects are superimposed or combined in the autotrack pitch and roll error signature that is used to evaluate the quasistatic variation.

\section{MBA Non-Thermal Distortion}

The on-orbit MBA non-thermal distortions effects can be classified as:

1) $1 \mathrm{~Hz}$ Oscillation

2) Yaw error induced by attitude control system

The $1 \mathrm{~Hz}$ Oscillation distortion affects all downlink beams and is caused by induced mechanical noise on the transmit main reflector. This mechanical noise is generated by the attitude control momentum wheel.

The yaw control errors are generated by poor convergence of the yaw estimator when it is in control of the $S / C$ attitude.

\section{$1 \mathrm{~Hz}$ Oscillation}

Figure 23, shows the distorted signal received by LET as a function of time. It can be seen from this signature that a $1 \mathrm{~Hz}$ sinusoidal variation is imposed on the receive signal. Figure 24, shows how the amplitude distortion changes with time of day.

Several experiments were conducted to measure amplitude of the oscillation. These experiments concluded that the $1 \mathrm{~Hz}$ oscillation was minimum $(<0.1 \mathrm{~dB})$ at beam center and becomes very strong $(>3.0 \mathrm{~dB})$ at beam edge. This result implies that the received amplitude variation is a function of the local MBA beam slope rate where the receive station is located within the beam. Stations located in contours with very high gain slope ( $>50.0 \mathrm{~dB} /$ degree) will experience a large amplitude variation than the stations located within the $3 \mathrm{~dB}$ contours. The amplitude was measured (peak-to-peak amplitude) to be $0.075^{\circ}$. 
Yaw Control Errors

The independent assessment of the causes for yaw errors are very difficult to determine from the available data base. The combined effect of yaw error (thermal and non-thermal) on beam pointing can be seen on the autotrack pitch and roll error signals (See Figure 25). The two S/C attitude (pitch, roll and yaw) control modes are the autotrack and the ESA systems. If the autotrack control is used, the $S / C$ rotates (yaw axis) around the Cleveland beam boresight. When the ESA control system is used, the $S / C$ rotates (yaw axis) around the subsatellite point. This implies that the effect of yaw error is larger when the ESA controls the S/C than when the autotrack system is in control. This effect of point of rotation between the two systems (ESA vs. Autotrack) can be seen by the received RF signal on the NGS (See Figure 25). In addition, environmental forces (magnetic storms, magnetic Earth field estimation, etc.) acting on the magnetic torquers induce large yaw errors. Figure 26, shows how the S/C yaw estimations compared with the exact sun sensor data as a function of time of the year. The exact yaw error is known only at the two sun windows (East and West). The exact yaw angles are used by the yaw estimator as boundary condition estimating and controlling the yaw for times outside the sun window.

\section{Compensation of ACTS MBA Thermal Distortions}

The compensation of thermal effects on the ACTS MBA beam pointing is achieved by using a combined autotrack system and a variable bias ESA control. This combination, over a 24 hour cycle, works very well for compensating the quasistatic variation that is produced by an unbiased ESA control system. Figure 27, shows the East receive autotrack error signals before and after compensation has been applied.

The impact on system performance using compensation is significant and can be summarized in the following three areas. First, the seasonal quasistatic effects on MBA beam pointing was minimized to $+/-0.01^{\circ}$ in pitch and $+/-0.01^{\circ}$ in roll (See Figure 30 ). Secondly, the effects on ESA attitude control instability over a $24 \mathrm{hr}$. cycle was also minimized. The signature on the autotrack shows a very steady response over the $24 \mathrm{hr}$. cycle, as opposed to using the ESA control.
Finally, we extend the stable time on the uplink West beams contours to $24 \mathrm{hr}$. and to $16 \mathrm{hrs}$. on the East beams (due to rapidly varying thermal events). The downlink beams required further compen-sation of the diurnal variation thermal effects. This effect is compensated by using the biax drive motor (azimuth and elevation) on the transmit main reflector. After the biax drive compensation is applied, the downlink West beams resulted in a stable time of $24 \mathrm{hrs}$. and $16 \mathrm{hrs}$. (because of rapidly varying events) on the East beams.

\section{Network Control Readiness Tests}

Two areas were emphasized in this demonstration of readiness:

1) Protocol performance (including network control

2) Spacecraft/Earth station link performance

These areas had previously been thoroughly tested, but without the effects of satellite stationkeeping, attitude control, thermal variations, range variations, a large VSAT network and rain attenuation.

\section{Network Control Performance}

Network control is provided by the Master Control Station (MCS) located at NASA Lewis Research Center. Four functions, performed by the MCS, have been identified as having significant impact on overall network control performance:

1) VSAT antenna alignment

2) VSAT acquisition and synchronization

3) Adaptive rain fade compensation

4) Demand Assigned Multiple

Assess (DAMA)

\section{VSAT Antenna Alignment}

VSAT antenna alignment is the process of "finding" the S/C downlink signal. To accomplish this, the $S / C$ must provide a signal to the VSAT location continuously for up to an hour without disrupting ongoing network opera The signal provided is a TDMA burst referred to as the Receive Acquisition Burst (RAB). It is a $24.9 \mu \mathrm{sec}$ burst transmitted by the S/C at the end of each $1 \mathrm{msec}$ TDMA frame. This "continuous" signal has a duty cycle of about $2.5 \%$. As a result of Reference Station operator control, the RAB is directed to dwell on the VSAT to be aligned for 90 
seconds. It may then move to another acquiring VSAT revisiting the aligning VSAT 5 times before stopping transmission.

Installers at the aligning VSAT site point the VSAT antenna to calculated S/C azimuth and elevation until the AGC voltage at a demodulator AGC test point is peaked. At this point, the antenna is locked in position.

Once on site azimuth and elevation references have been established, this antenna alignment is routinely accomplished without disrupting normal network operations and typically in a half hour or less.

\section{VSAT Acquisition and Synchronization}

VSAT acquisition is the process of synchronizing to network protocols, to receive and transmit frame timing and to superframe timing. During a normal week of operations, this process may be executed as seldom as once a week or as often as several times a day per VSAT.

Design analyses of the acquisition and synchronization processes predicted that given the S/C remains within its stationkeeping design limits of $+.05^{\circ} \mathrm{E} / \mathrm{W}$ and $+.05^{\circ} \mathrm{N} / \mathrm{S}$, and given the VSAT location is accurate to within 50 meters, an Acquisition Search Range of 95 slots will result in successful acquisition of all VSATs located within the continental boundaries of the U.S.

S/C tracking data indicates that the S/C stays well within its design limits. However, using Global Positioning Satellite (GPS) receivers, VSATs can be located with an accuracy of only about 100 meters. In spite of this uncertainty and using an Acquisition Search Range of 95 slots, successful acquisition of VSATs has been demonstrated at locations spread across the continental U.S., as well as in Alaska, Hawaii, Ecuador and Columbia, S.A. Furthermore, successful acquisition is likely if the VSAT latitude and longitude are known within $.05^{\circ}$.

An Acquisition Search Range of 95 slots results in an Acquisition Window in the uplink code portion of the frame of 274 slots. This is a relatively large part of the coded frame. Since the acquisition process shares the coded frame with the rain fade compensation process, it is possible that an acquiring VSAT could preclude another VSAT's transition to coded operation during a rain event. This situation can be considerably improved if the Acquisition Search Range can be reduced; e.g., to a value of 25 or less. This would reduce the Acquisition Window to 134 slots. Testing is ongoing to establish the minimum workable Acquisition Search Range.

\section{Adaptive Rain Fade Compensation}

Adaptive rain fade compensation is the process whereby a VSAT's bit error rate performance is automatically enhanced during a period of signal loss due to rain attenuation. In clear sky conditions, ACTS RF links are designed to operate with $5 \mathrm{~dB}$ and $3 \mathrm{~dB}$ of uplink and downlink margin, respectively. With the uplink and downlink margins reduced to zero, the links are designed to provide an uplink or downlink bit error rate of 5E-7. Rain fade compensation in the amount of an added $10 \mathrm{~dB}$ of margin on each link is provided by reducing the burst rate by half and invoking rate onehalf forward error correction coding. Added to the above clear sky margin, the total uplink margin is $15 \mathrm{~dB}$ and the total downlink margin is $13 \mathrm{~dB}$. The compensation is adaptive since it is only invoked when needed. Additionally, the invoking of coded vs. uncoded operation must be timely; i.e., it must accommodate a rain fade rate of $.25 \mathrm{~dB}$ per second, must have no impact on throughput and must be "hitless" in the transition from one to the other.

Data has shown that the ACTS adaptive rain fade compensation protocols successfully provide enhanced bit error rate performance automatically as needed. The transitions from uncoded to coded operation and back are accomplished without error. Considerably more testing is required before the performance can be fully evaluated against rain statistics. This testing is ongoing.

\section{Demand Assigned Multiple Access}

Demand assigned Multiple Access (DAMA) is the process that enables unused S/C capacity to be shared by all network VSATs, that enables full mesh connectivity between all network VSAT that enables voice and wideband data circuits to be connected and disconnected on demand. Shared S/C capacity means that unused circuits can be shifted from a VSAT that is not using it to a VSAT that needs it. Full mesh connectivity means that any VSAT can communicate directly with any and all others. On-demand voice or data circuits 
means that a circuit is created in the network by the MCS only when needed and torn down when no longer needed.

Call set-up for voice is accomplished similarly to terrestrial call set-up; i.e., using the North American Dialing Plan. Wideband data circuit set-up is accomplished using a touch-tone telephone handset connected to the network and a string of dial digits as shown below.

\section{Circuit Connect:}

Using a handset on a VSAT, the digit string $2201000 * 24 * 12 * 1100 *$ is dialed where:

\section{* Delimiter}

20 S/C duplex data call connect feature code

1000 Logical port number of the 1st channel of the origination data trunk

24 Number of channels in the wideband circuit

12 Destination VSAT number

1100 Logical port number of the $1 \mathrm{st}$ channel of the destination data trunk

The MCS identifies the idle channels in the VSAT's burst and transmits new burst assignments to each VSAT affected by the shift in capacity.

After all VSAT assignments have been updated, all are switched in unison to the new burst time plan.

With the switch to the new burst time plan, the 24 channel circuit is completed and ready for use.

\section{Circuit Disconnect:}

Using a handset on the VSAT, the digit string $401000 *$ is dialed.

40 S/C duplex data call disconnect feature code

1000 Logical port number of the 1st channel of the origination (or destination) data trunk

Voice call set-up protocols have been successfully demonstrated throughout on-orbit testing and operations. Measured voice call set times have averaged 11 seconds from the last digit dialed to the first ringback. Voice quality is generally as good as, or better than terrestrial quality. Data circuit set-up protocols have also been successfully demonstrated with set-up times between 6 and 12 seconds.

\section{CONCLUSION}

The on-orbit tests conducted after launch showed that the payload and bus performance was in good agreement with the ground test results. Since then, there have been no failures of the equipment on-orbit.

Analysis and on-orbit measurements found the MBA thermal distortions to be periodic. This periodicity makes it possible to predict the effects of the uncompensated rapidly varying events on system performance as a function of time of the day and time of the year. The compensation of thermal effects makes the West beams $100 \%$ stable, and East beams about $75 \%$ stable. Table IV, describes the system impact with, and without, compensation for ground stations within the $3 \mathrm{~dB}$ beam contour.

Future communications satellites using multibeam reflector systems at $\mathrm{Ka}$ band, or higher, frequencies employing gridded reflector structures (cause for rapidly varying thermal events) and materials like Aztroquartz (with a high coefficient of thermal expansion) should provide a sunshade to avoid thermal distortion.

The mechanical oscillations (nonthermal distortions) on the ACTS MBA are very difficult to compensate. The $1 \mathrm{~Hz}$ oscillations occur at the resonant frequency of the antenna, which was kept unduly low by weight and volume constraints on the MBA design.

The yaw control system can affect the beam pointing drastically especially for those ground stations located away from either sub-satellite longitude (ESA), or autotrack boresight. Future spot beam spacecraft systems should make yaw estimation a primary concern in their design goals. The data collected $(>365$ days) on the ACTS yaw estimator shows the yaw error is very sensitive to the environmental changes and spacecraft mechanical noises. This makes the yaw estimation process during times outside the sun window very challenging.

The multibeam antenna on-orbit test measurement found the MBA performance to be well within the expected range, and the transmit and receive beam optimization procedures were successfully executed. In general, the on-orbit 
MBA measurements have shown that all design parameters have been met and that pointing requirements have been achieved.

In reference to the VSAT network, voice call set-up protocols have been successfully demonstrated throughout on-orbit testing and operations. Measured voice call set-up times have averaged 11 seconds from the last digit dialed to the first ringback. Voice quality is generally as good as, or better than, terrestrial quality. Data circuit set-up protocols have also been successfully demonstrated with set-up times between 6 and 12 seconds.

In conclusion, the ACTS system has met its design objectives. It has provided the impetus for the current demand of spectrum at $\mathrm{Ka}$ band, and has proven that on-board processing is feasible and works well.

The system has also allowed ground measurements of beam stability that had been very difficult to make with large coverage area satellites, thus advancing the state-of-the-art in satellite pointing and attitude control. 


\section{SPACECRAFT}

Customer . . . . . . . . . . . . NASA Lewis Research Center

Type . . . . . . . . . . . . . 3-Axis Stabilized Communications Technology Satellite

Application. . . . . . . . . . Testbed of New Technology Applications Available to U.S.

Launch Vehicle ............. STS/TOS

Experimenters Free of Charge

Launch Weight ........... $6108 \mathrm{lbs}$.

Orbit Position . . . . . . . . Geosynchronous, Equatorial 100 West

Design Life. . . . . . . . . 4 years

\section{Electrical Power Distribution}

Solar Array Output. . . . . . . . 1418 Watts (4 Years)

Battery System ........... 2 NiCd Batteries of 19 AH Each. No Payload Operation During Eclipse

Power Bus. . . . . . . . . $35.5( \pm 0.5)$ Volts with Full Array Illumination

Propulsion and Orbit Control

Design.

Blowdown Hydrazine System with Redundant Thrusters and Four

Tanks

Propellant. . . . . . . . . . $580 \mathrm{lbs}$

Thrusters .......... $16(0.2,0.5$, and $1.0 \mathrm{lbf})$

Stationkeeping. .......... $\pm 0.05^{\circ}$

\section{Structure and Thermal}

Structure . . . . . . . . . Length: 80"; Width: 84"; Depth: 75"

Solar Array. . . . . . . . . . . With Yoke, 46.9' Tip-to-Tip

Antenna Assembly. . . . . . . . Height: 116" Above Antenna Panel; Width: $29.9^{\prime}$ Deployed

Thermal Control ........... Passive Temperature Control: Blankets and OSR;

Attitude Control

Active Temperature Control: Solid State Controllers and Heaters

Transfer Orbit Control ....... Autonomous Nutation Control During Spin. Initial Pointing

Provided by TOS Stage

On-Orbit Control . . . . . . . . . 3-Axis Stabilized via Earth and Sun Sensor and Momentum Wheel.

Pointing Accuracy $\ldots . . . . . . . \quad$ Autotrack Ref. Used During Communications Experiment Periods

Roll, $0.25^{\circ}$ Yaw Using Earth Sensor

Offset Pointing Control $\ldots \ldots \ldots \pm 6^{\circ}$ Pitch, $\pm 2^{\circ}$ Roll

Command, Ranging and Telemetry

Command Frequency. . . . . . . Ka-Band; C-Band Backup and Transfer Orbit

Command Rate. ........... 100 pps FSK for Bus Functions; 5000 pps SGLS for Payload

Command Capacity. . . . . . . . 379 Low Rate Discretes; 3 Serial Low Rate Data Streams: 256

Telemetry Frequency . High Rate Discretes; 3 Serial High Rate Data Streams

Transfer Orbit

Telemetry Format . . . . . . . . . 8 Bits/Words; 256 Words/Minor Frame; 25 Minor Frames/Major

Frame; 1024 bps

Telemetry Capacity . . . . . . . . 312 Bilevel Words; 364 Analog Words; 6 Serial Words; Dwell

Tracking Frequency . Capability on Any Analog, Bilevel or Serial Word

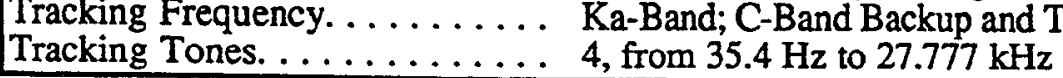

Table I - Spacecraft Bus Specifications. 


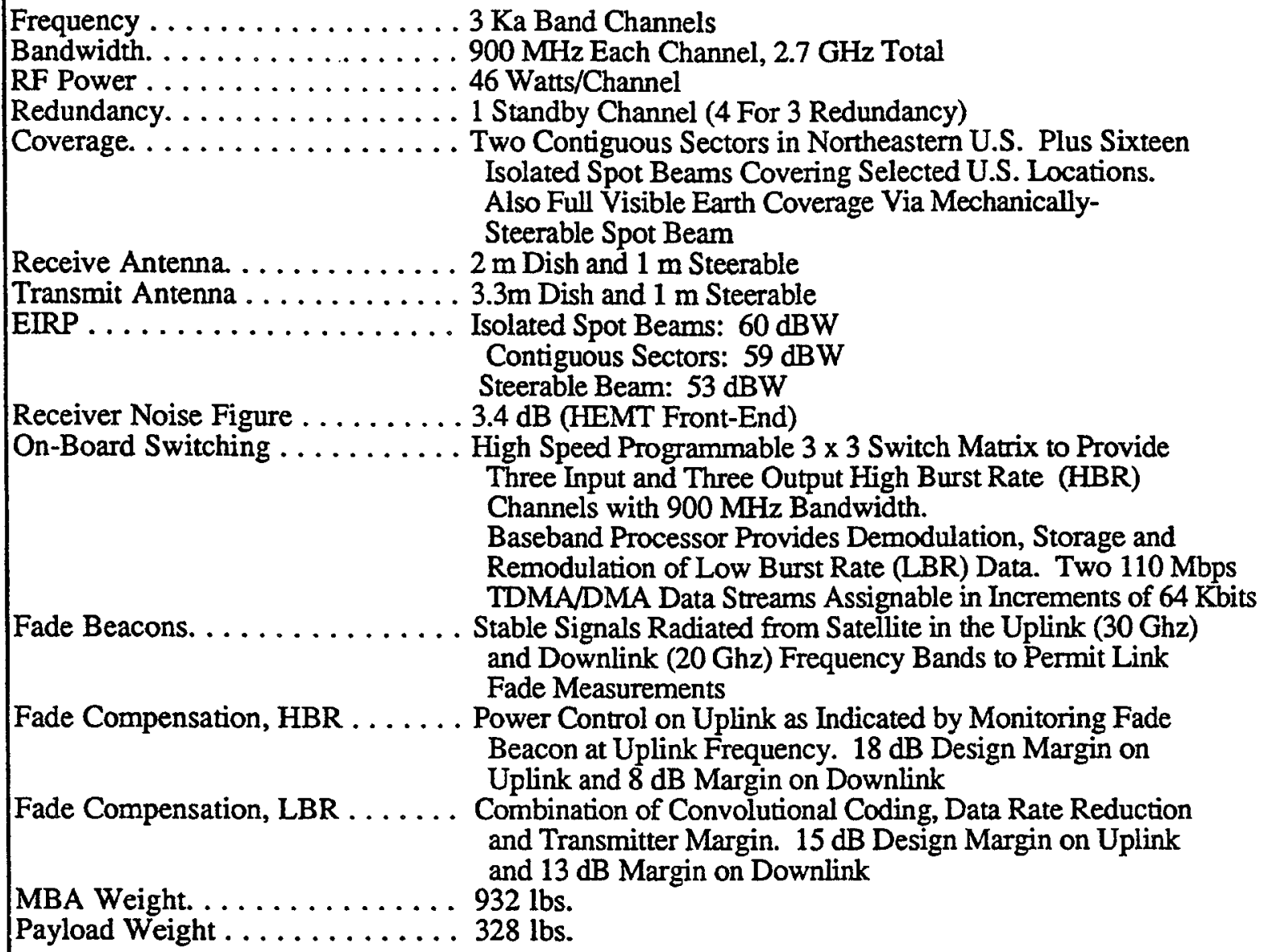

Table II - MBA and Communications Payload Specifications.

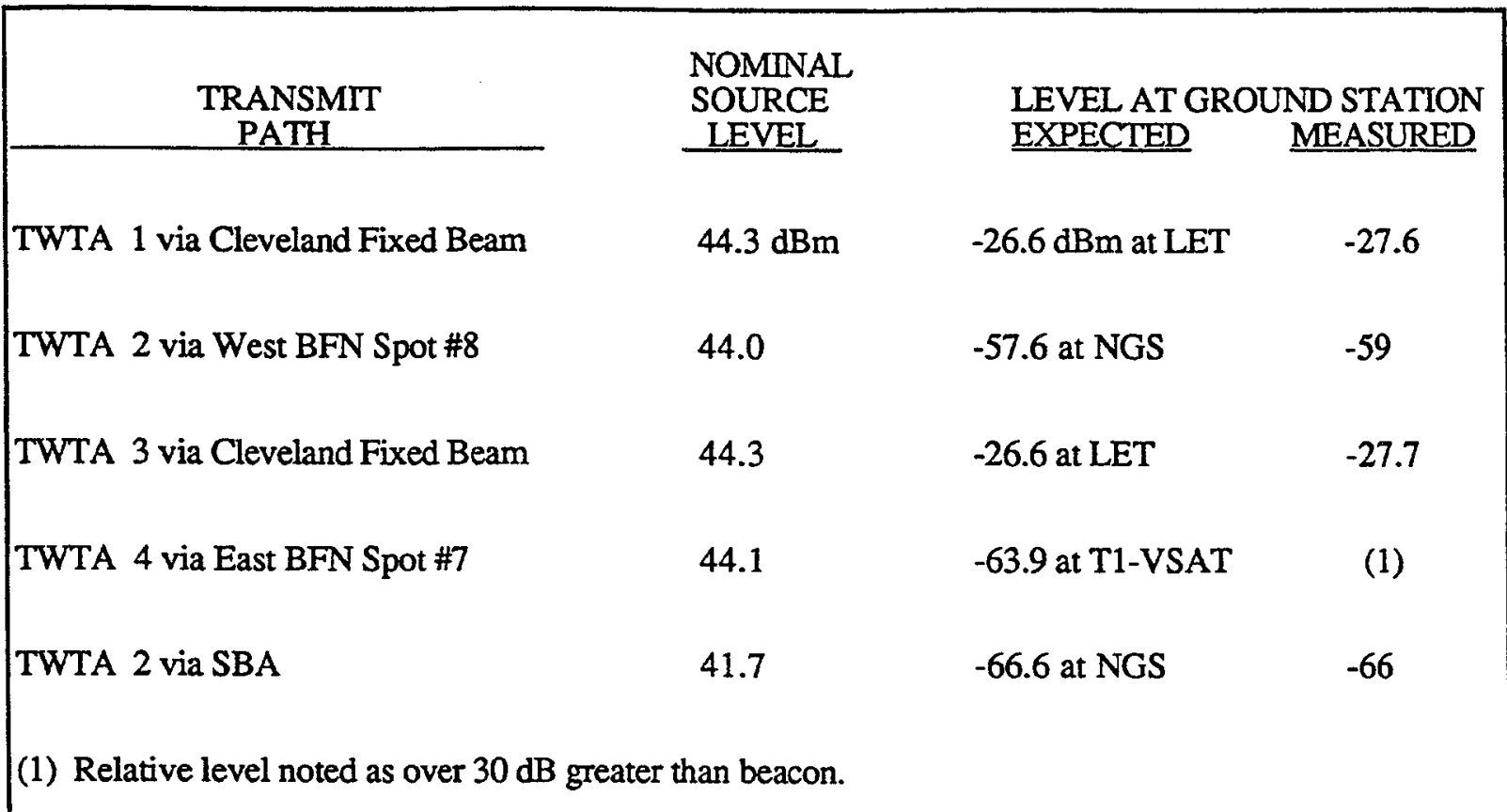

Table III - ACTS Downlink Performance. 


\begin{tabular}{|l|c|c|c|c|l|}
\hline \multicolumn{1}{|c|}{ TYPE } & BEAM & MAGNITUDE & AXIS & DURATION & $\begin{array}{l}\text { OPERATIONAL } \\
\text { IMPACT }\end{array}$ \\
\hline $\begin{array}{l}\text { Rapidly Varying } \\
\text { (PR \#291) }\end{array}$ & $\mathrm{E}$ & Less than 0.1 & $\mathrm{R}$ & $\begin{array}{c}\text { Less than an } \\
\text { hour }\end{array}$ & $\begin{array}{l}\text { Short term impact } \\
\text { on marginal station. } \\
\text { Use ESA control } \\
\text { during event to } \\
\text { minimize impact }\end{array}$ \\
\hline $\begin{array}{l}\text { Diurnal Variation } \\
\text { (PR \#237) }\end{array}$ & $\mathrm{E} \& \mathrm{~W}$ & $0.2^{\circ}$ & $\mathrm{P}$ & 12 hours/event & $\begin{array}{l}\text { Significant signal } \\
\text { variation, can crash } \\
\text { stations. Use Biax } \\
\text { drive to compensate }\end{array}$ \\
\hline $\begin{array}{l}\text { Quasistatic } \\
\text { (PR \#292) }\end{array}$ & $\mathrm{E} \mathrm{\&} \mathrm{W}$ & $\pm .04^{\circ}$ & $\mathrm{P}$ & 14 days & $\begin{array}{l}\text { Totally compensated } \\
\text { by Autotrack }\end{array}$ \\
\hline $\begin{array}{l}\text { Vibration } \\
\text { Tx }\end{array}$ & Tx Ant. & $\pm .012^{\circ}$ & $\mathrm{P}$ & One Hz & Generally negligible \\
\hline
\end{tabular}

Table IV - ACTS MBA RF Beam Pointing Variations. 


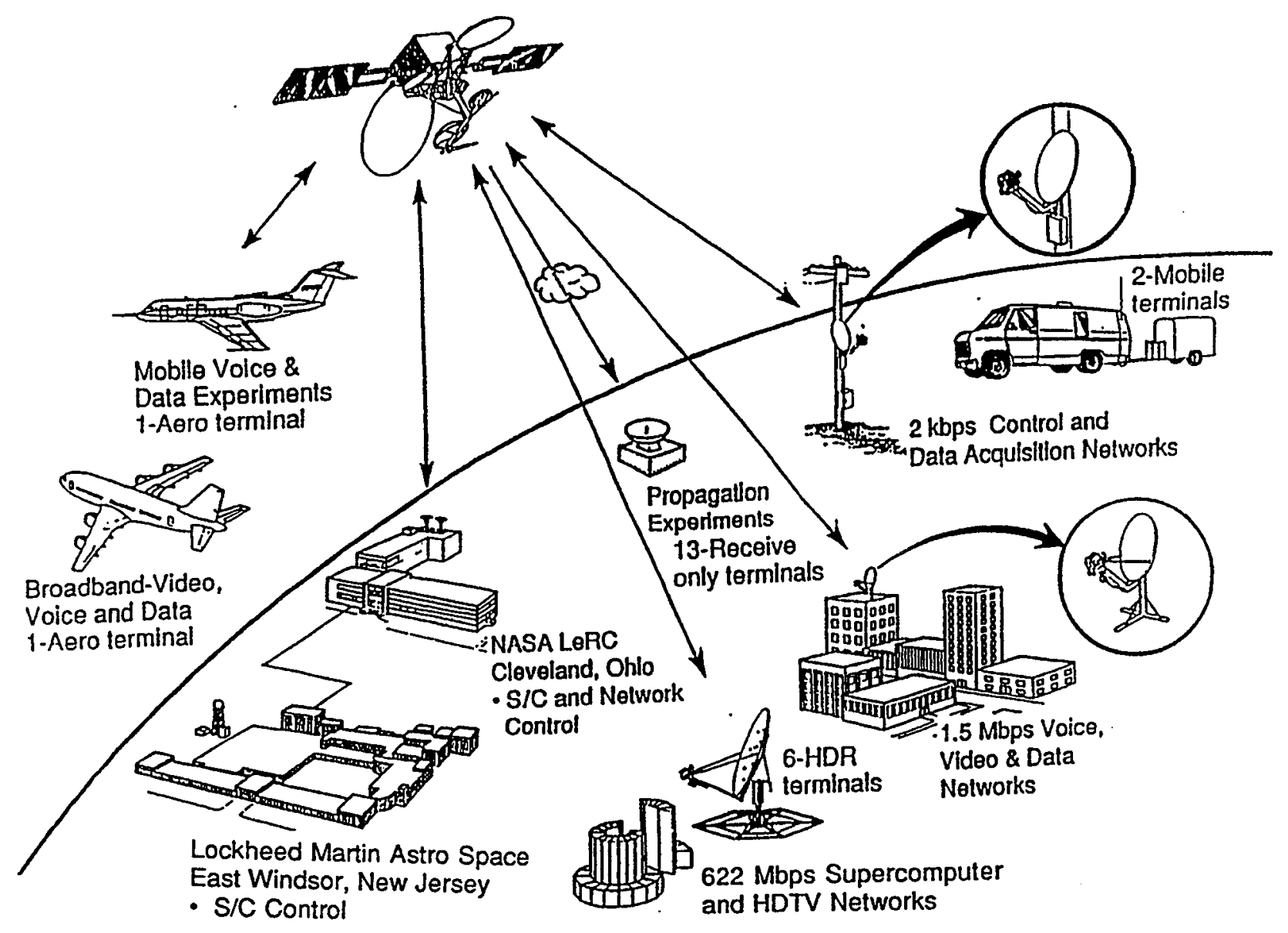

Figure 1. The ACTS System.

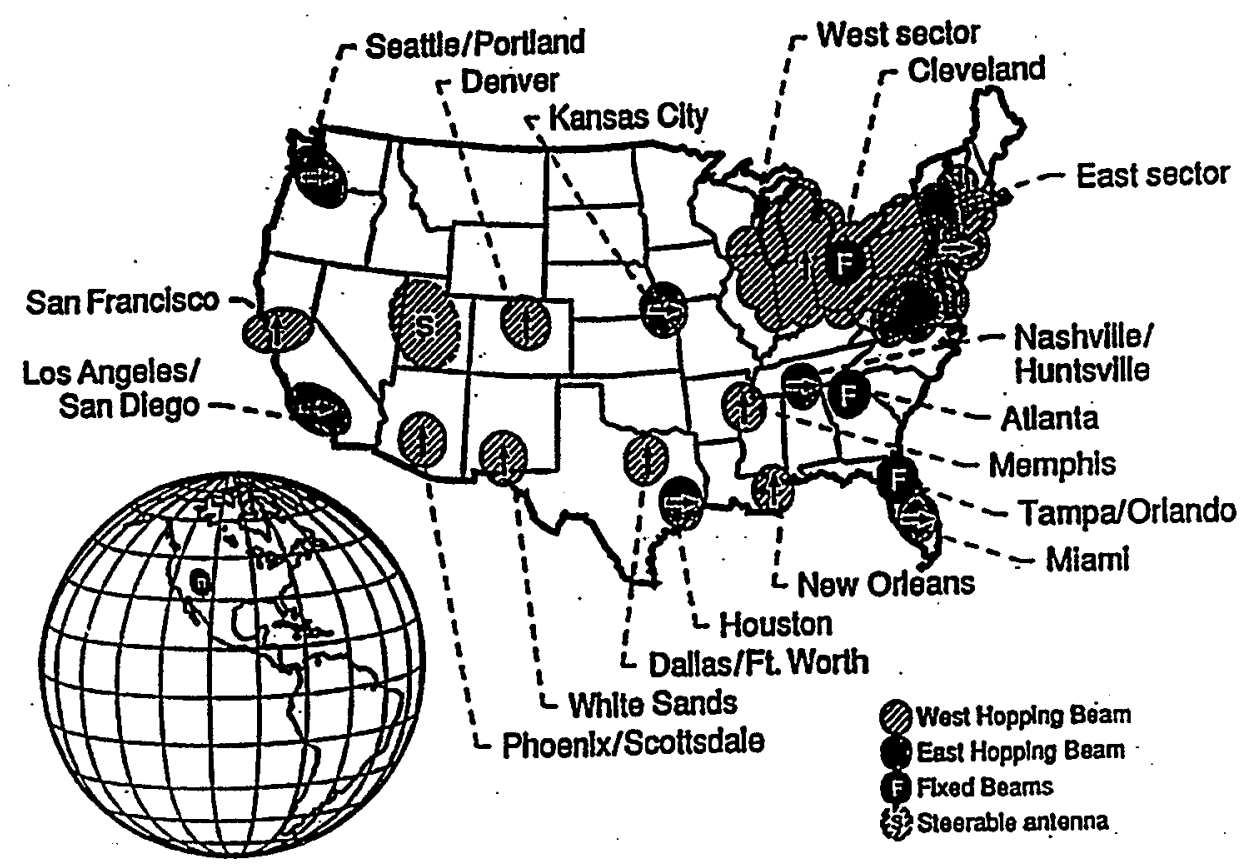

Figure 2 The ACTS Hopping Spot Beam and Steerable Antenna Coverage. 


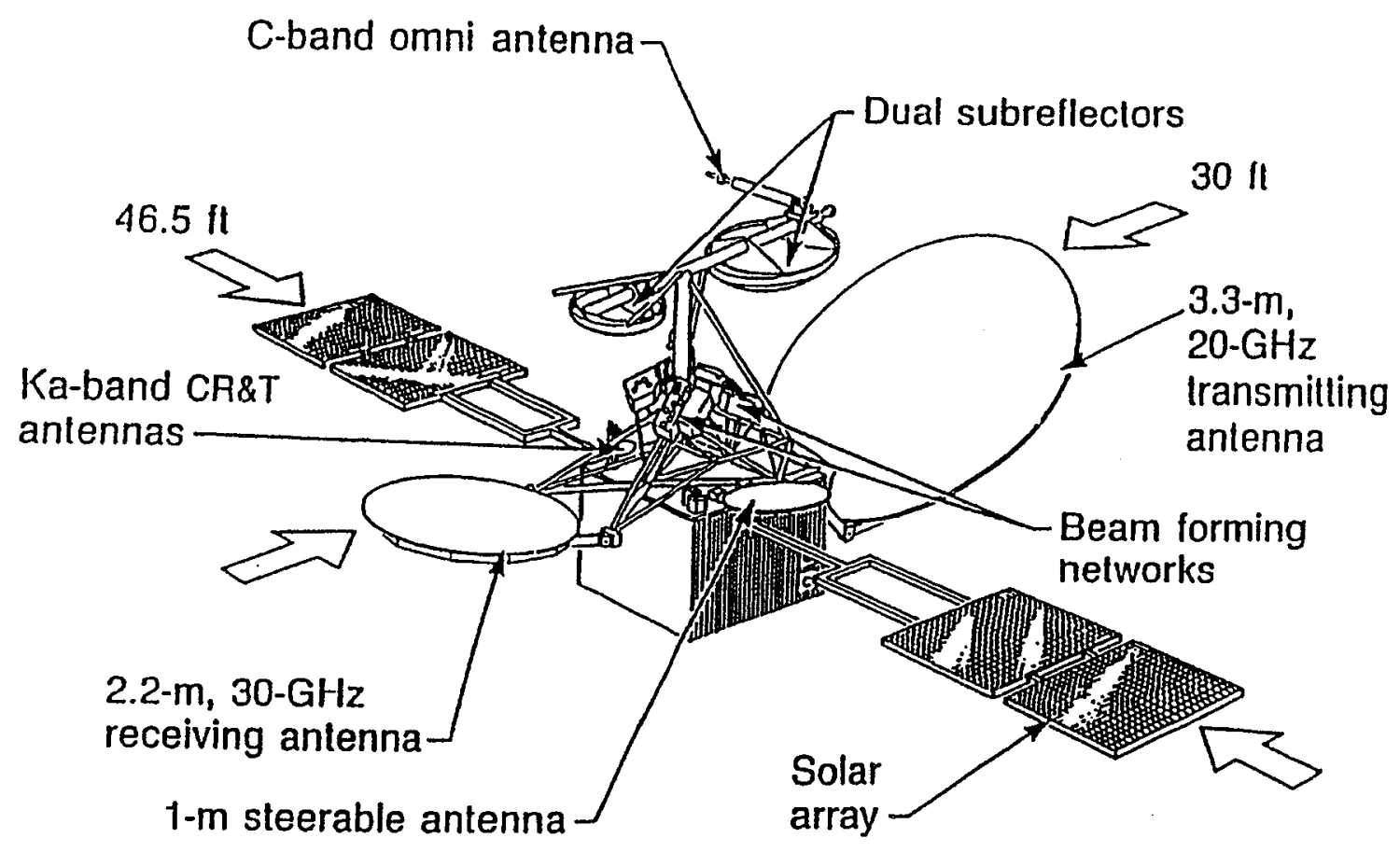

Figure 3. ACTS Spacecraft Configuration.

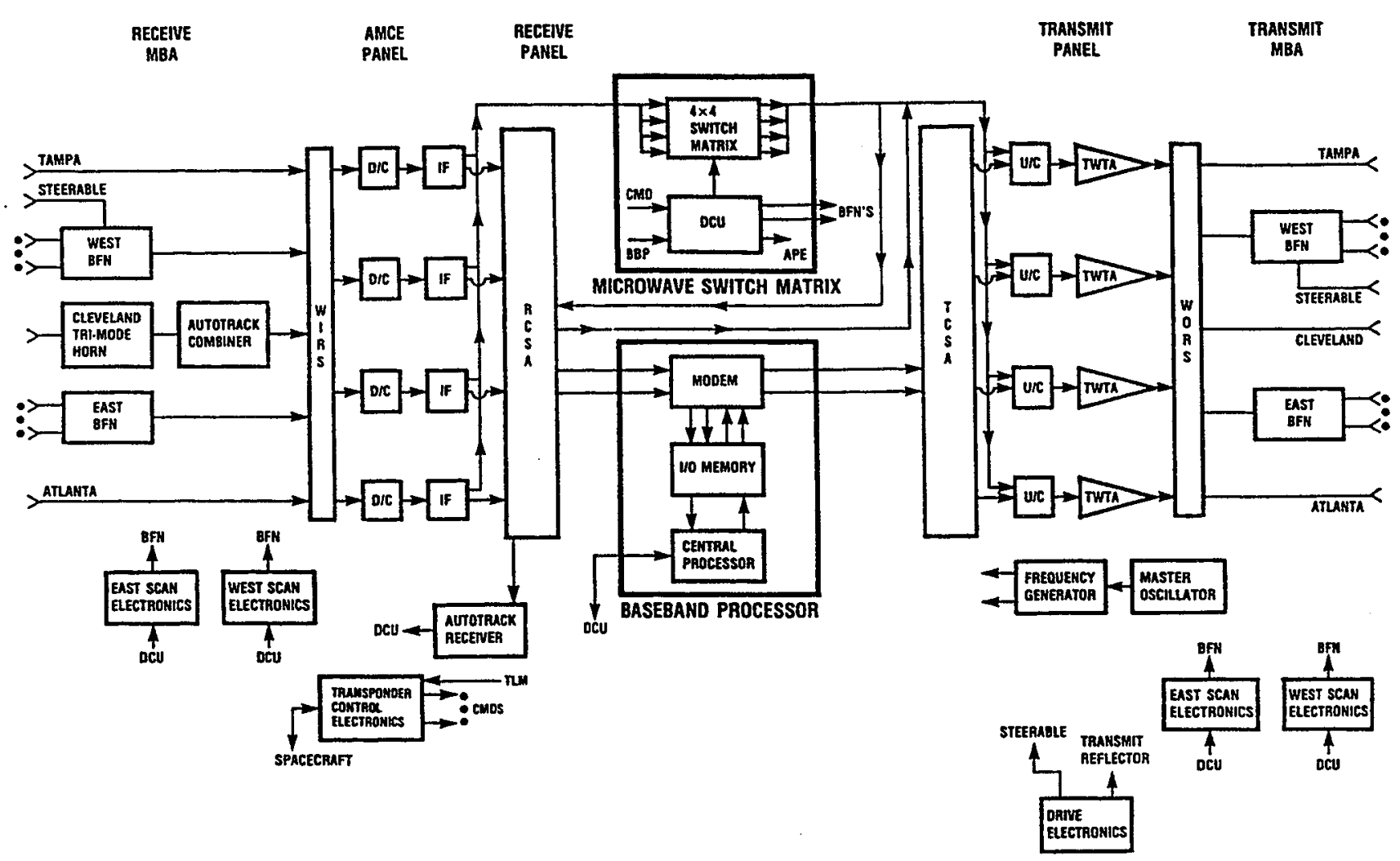

Figure 4. Block Diagram of the ACTS Communications Payload. 


\section{ACTS MULTIBEAM ANTENNA OFFSET CASSEGRAIN REFLECTOR ANTENNA GEOMETRY}

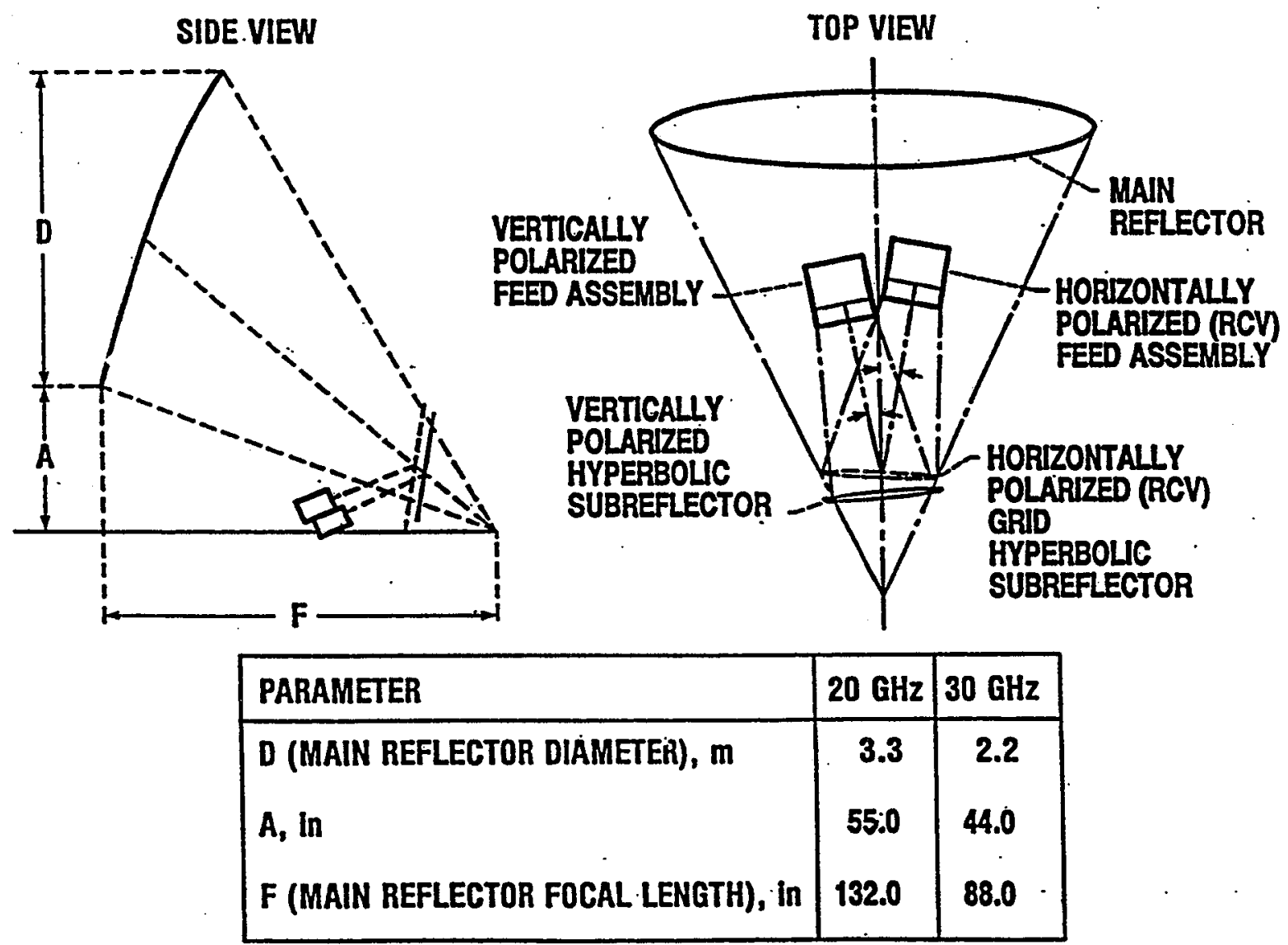

Figure 5 The ACTS Multibeam Antenna Geometry. 


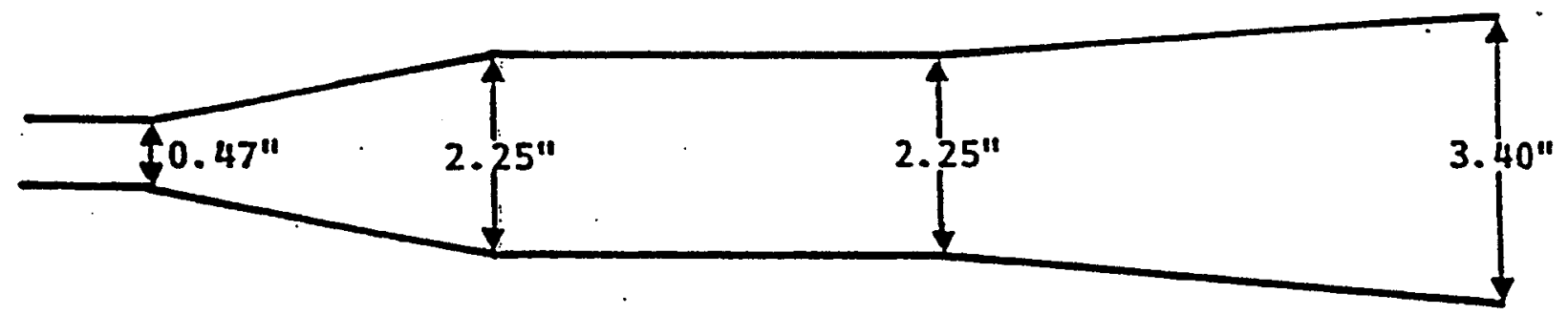

Figure 6 Transmit Trunking Horn
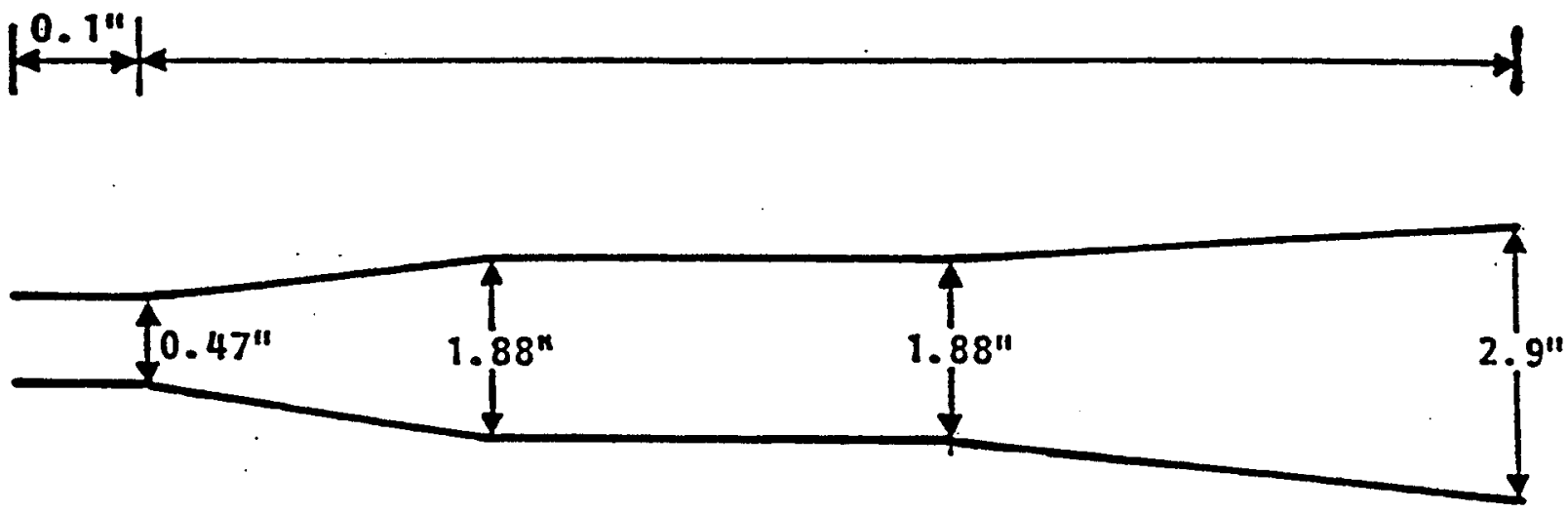

Figure 7 Transmit Scan Spot Horn

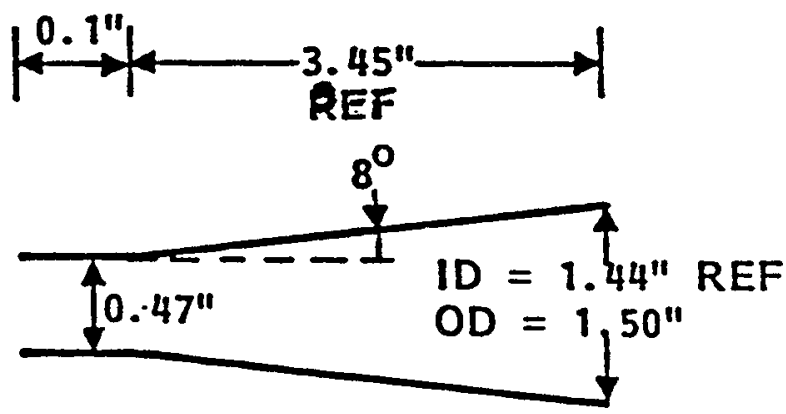

Figure 8 Transmit Scan Array Horn 

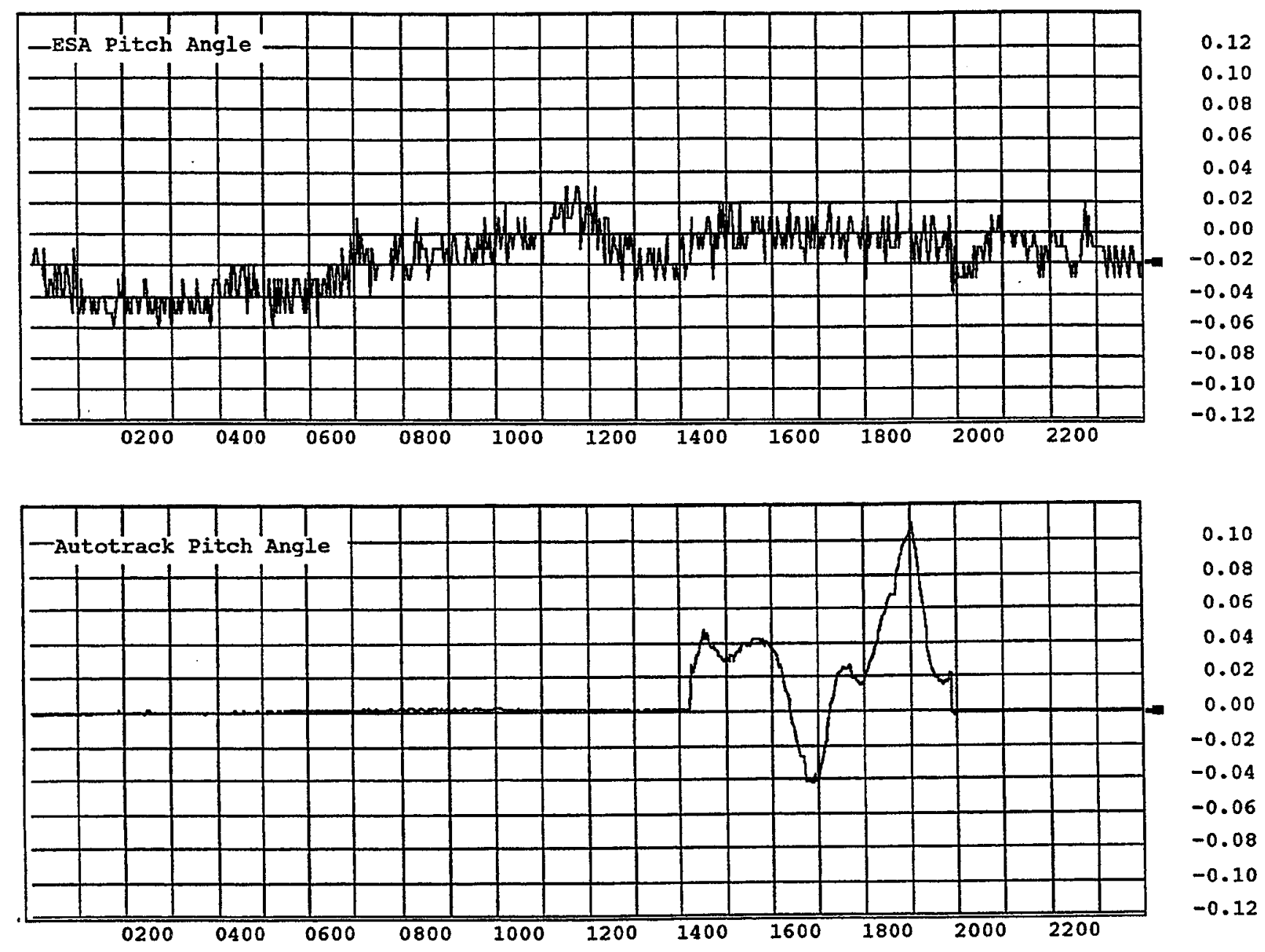

Figure 9. Typical pitch angle plot. Pitch is controlled by the ESA during the period of the Autotrack disturbance. 

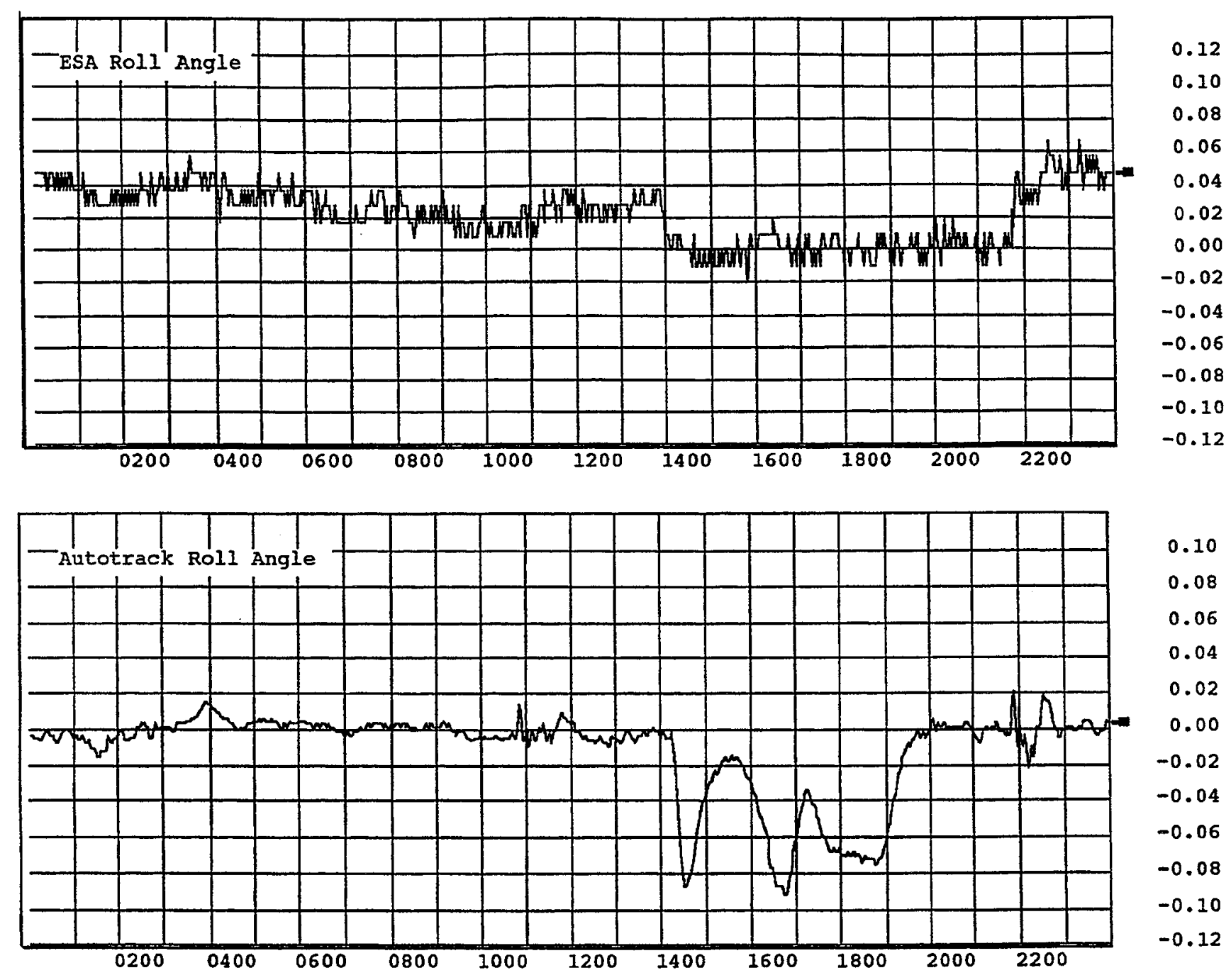

Figure 10. Typical roll angle plot. Roll is controlled by the ESA during the period of the Autotrack disturbance. 


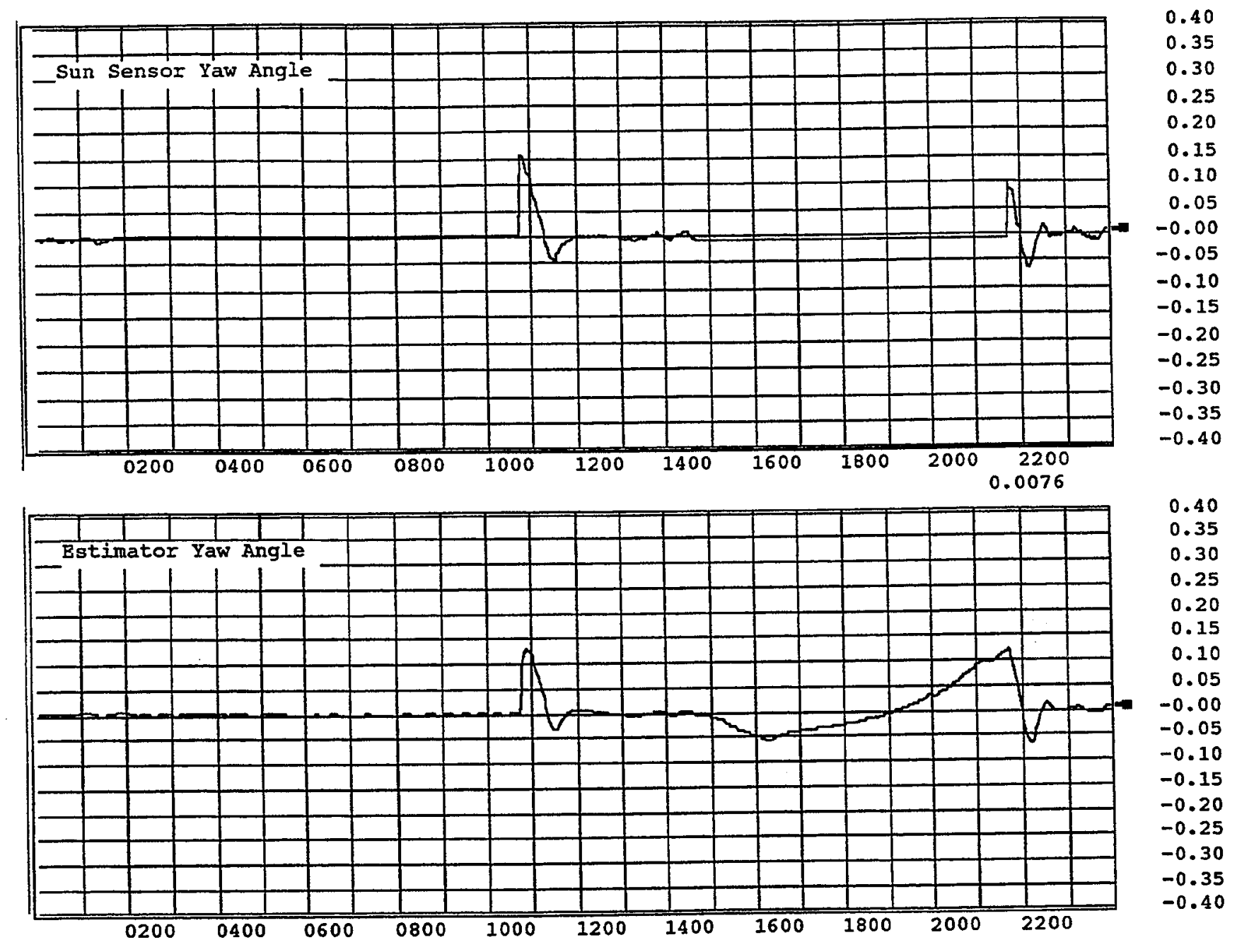

Figure 11. Typical yaw angle. The buildup of yaw error when the estimator is in control is quickly reduced when the sun sensors are activated. 


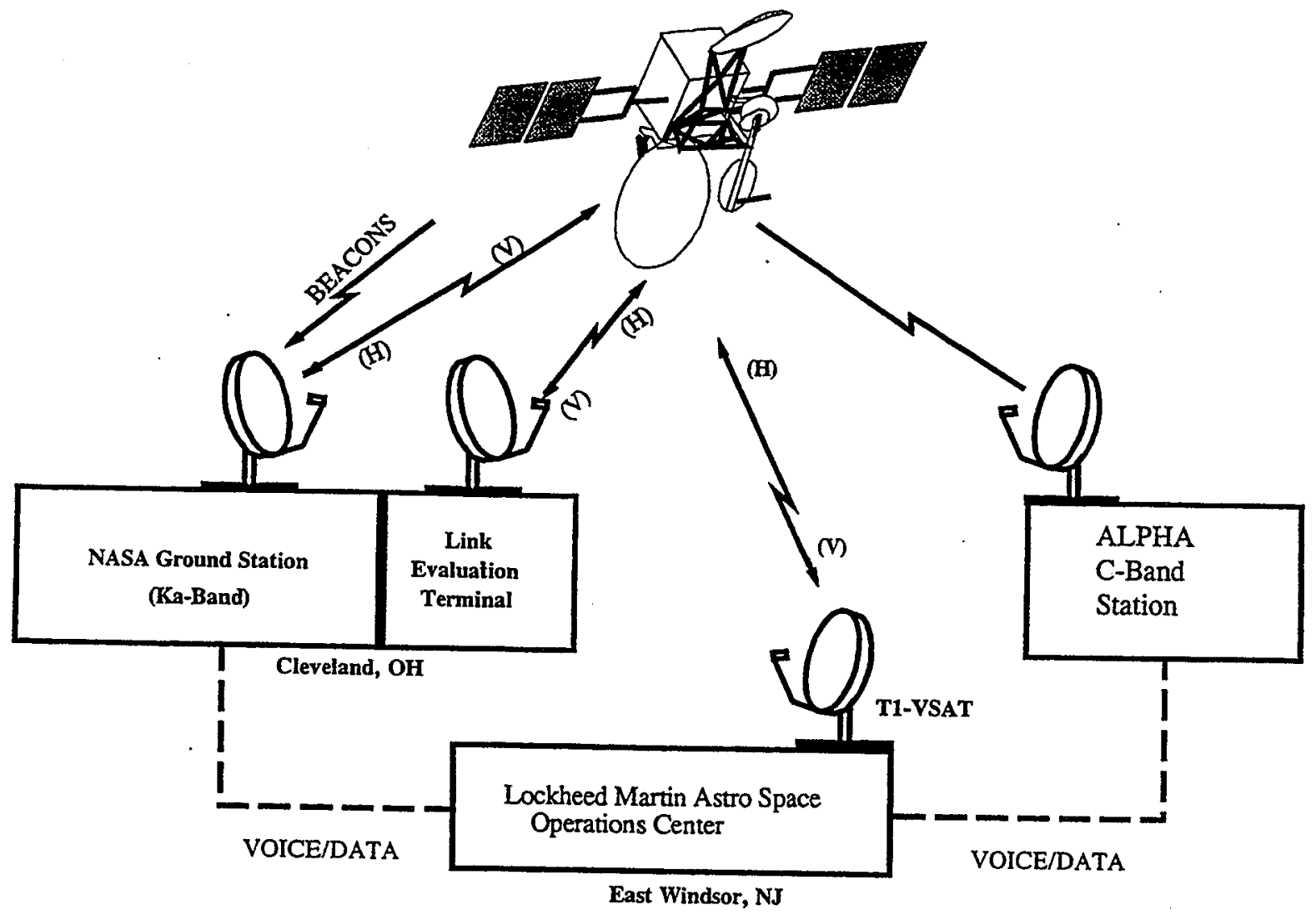

Figure 12. Ground stations used in initial on-orbit checkout of the ACTS satellite. 

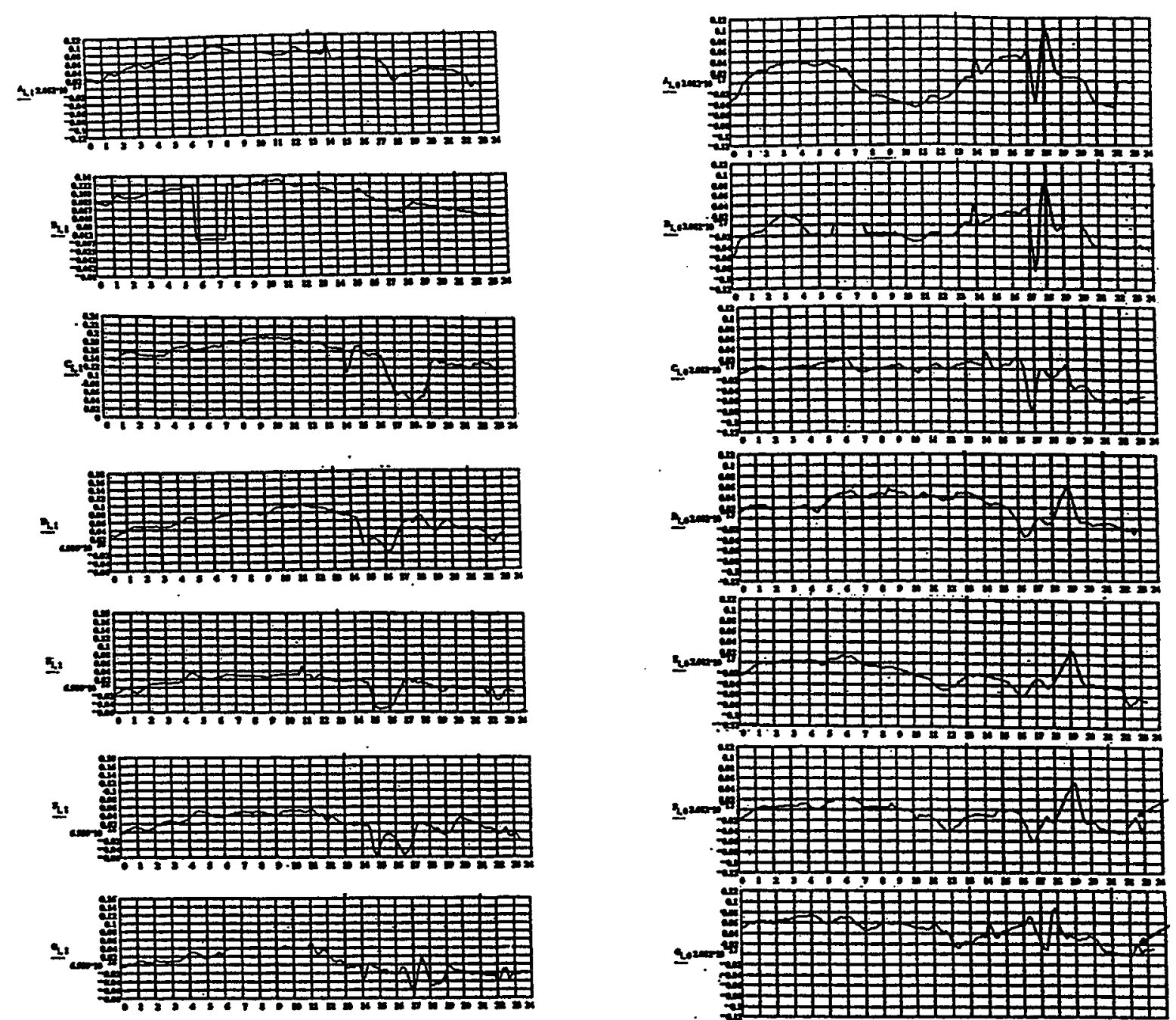


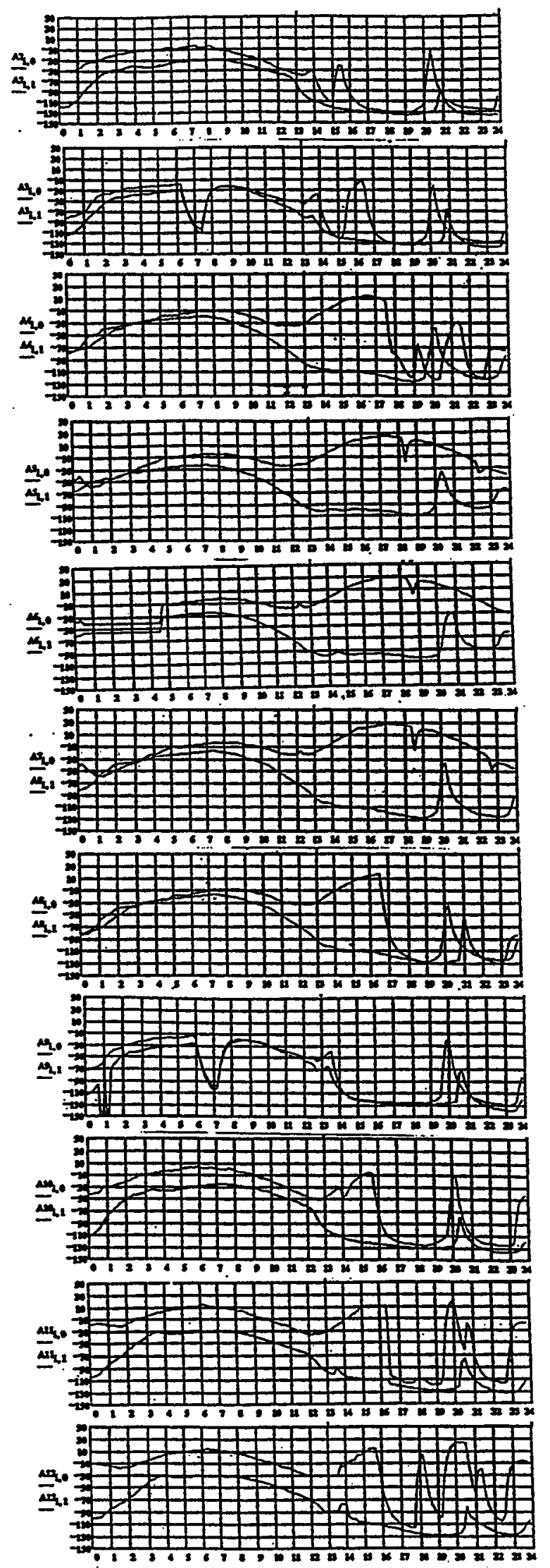

Figure 14 Receive front sub-reflector temperature profile as a function of time of the year. 

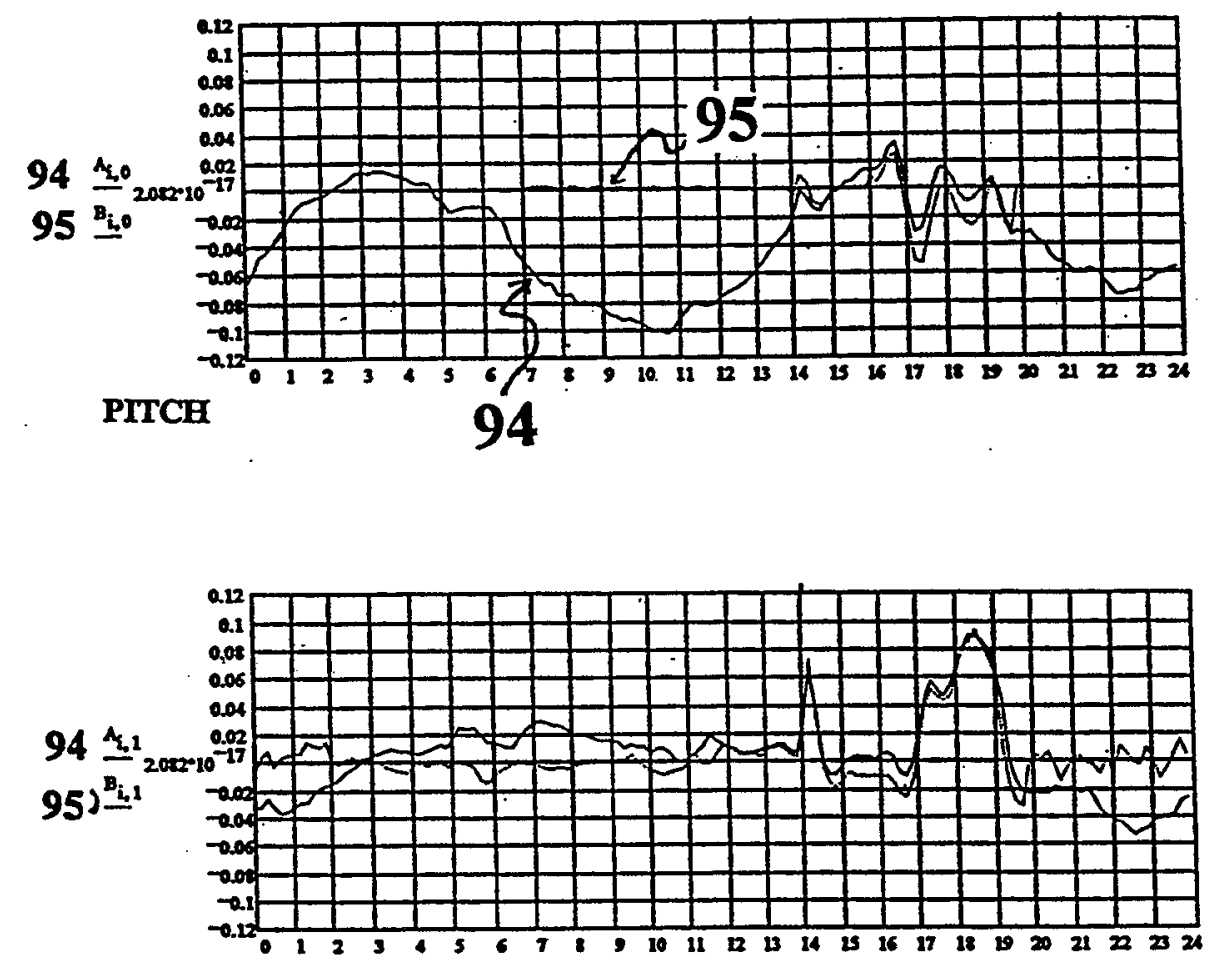

ROLL

Figure 15 Receive front sub-reflector pitch and roll error signatures for January 1994 and January 1995. 

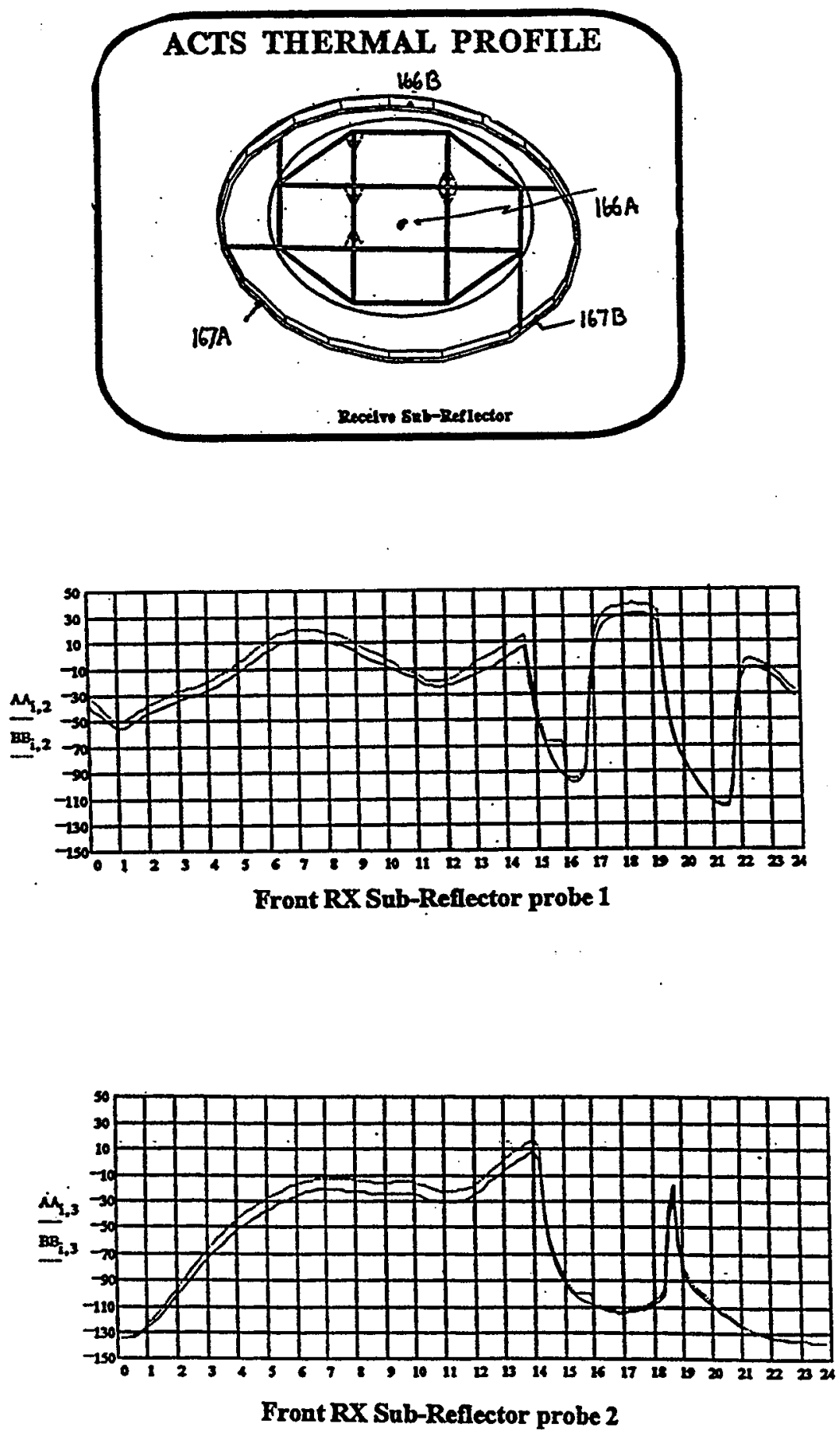

Figure 16 Receive front sub-reflector temperature profile for January 1994 and January 1995. 


\section{Winter Solstice 11:30 (s/c)}

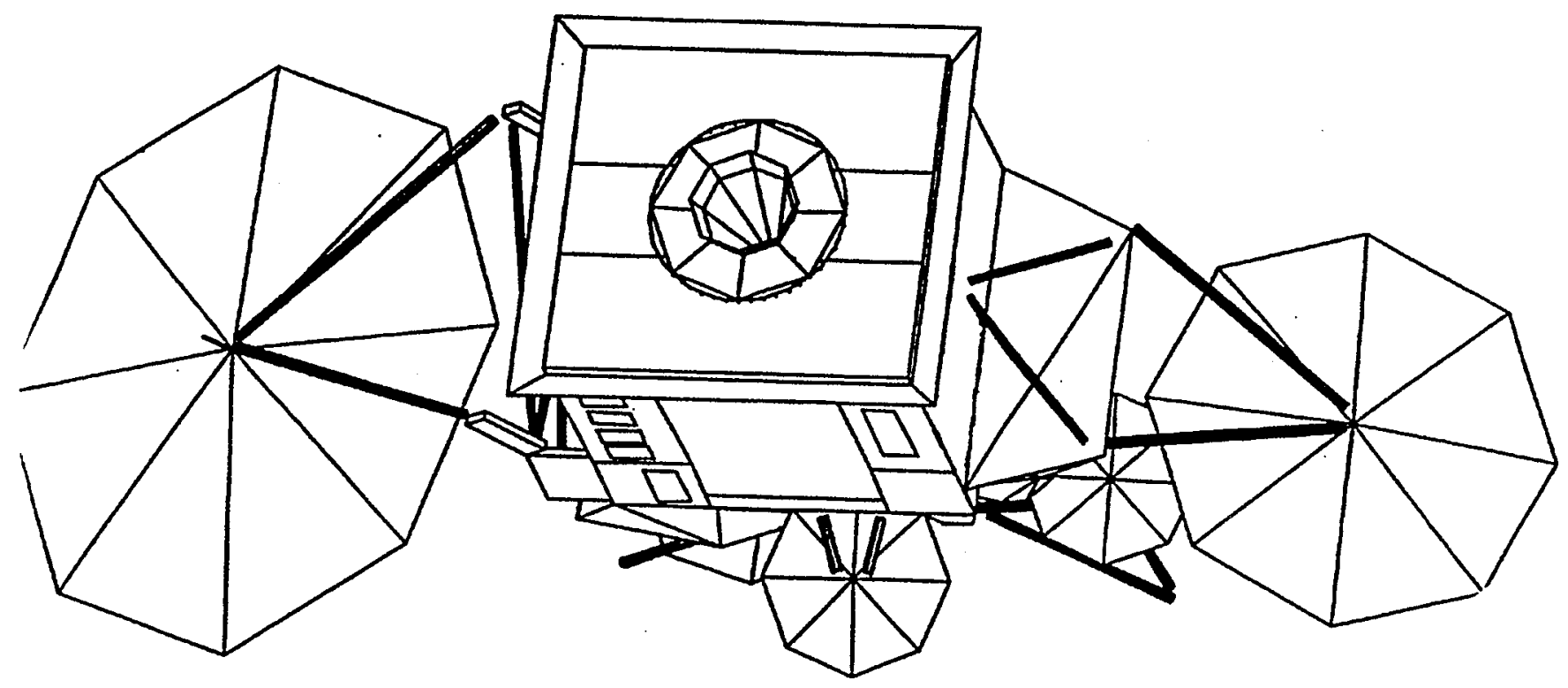

Figure 17 Sub-reflector orientation and shadowing relative to the sun (snapshot). 


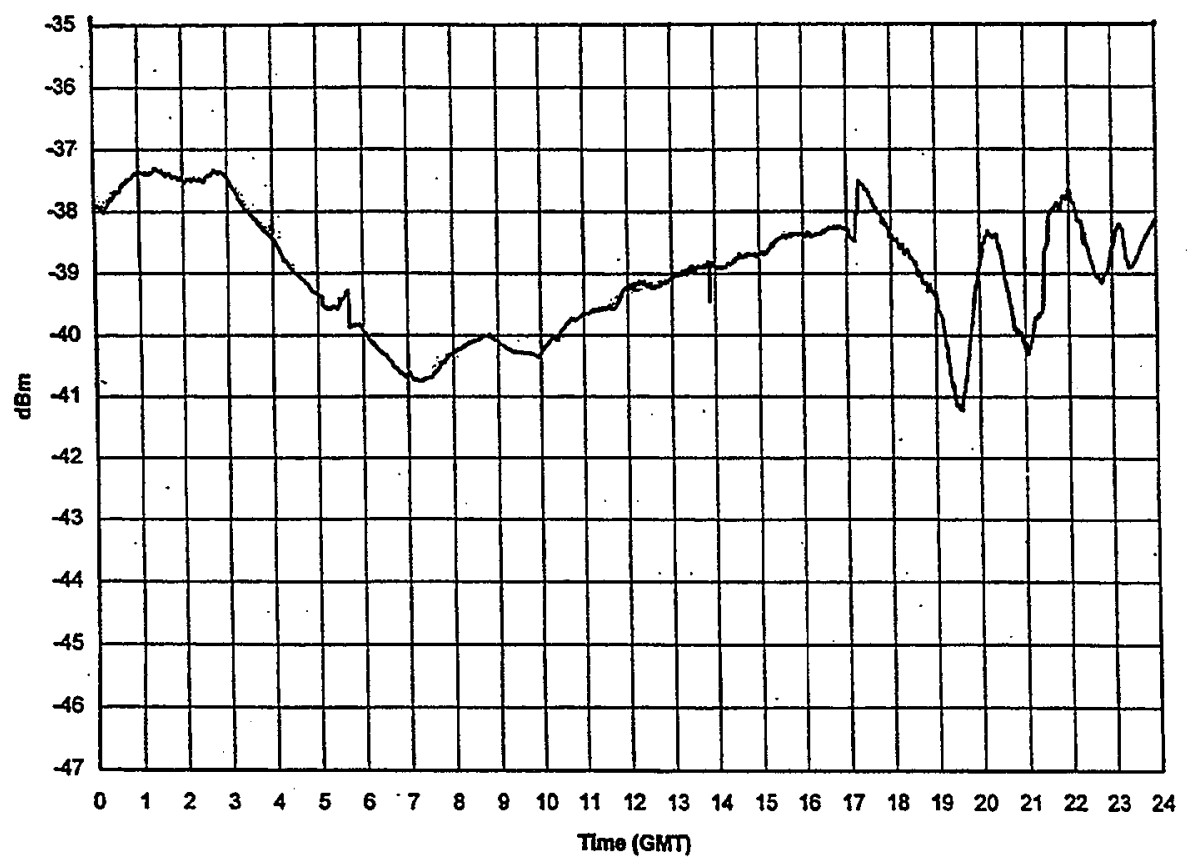

Figure 18 Typical Link Evaluation Terminal (LET) received signal (Cleveland Fixed Downlink Beam- East) as a function of time of day.

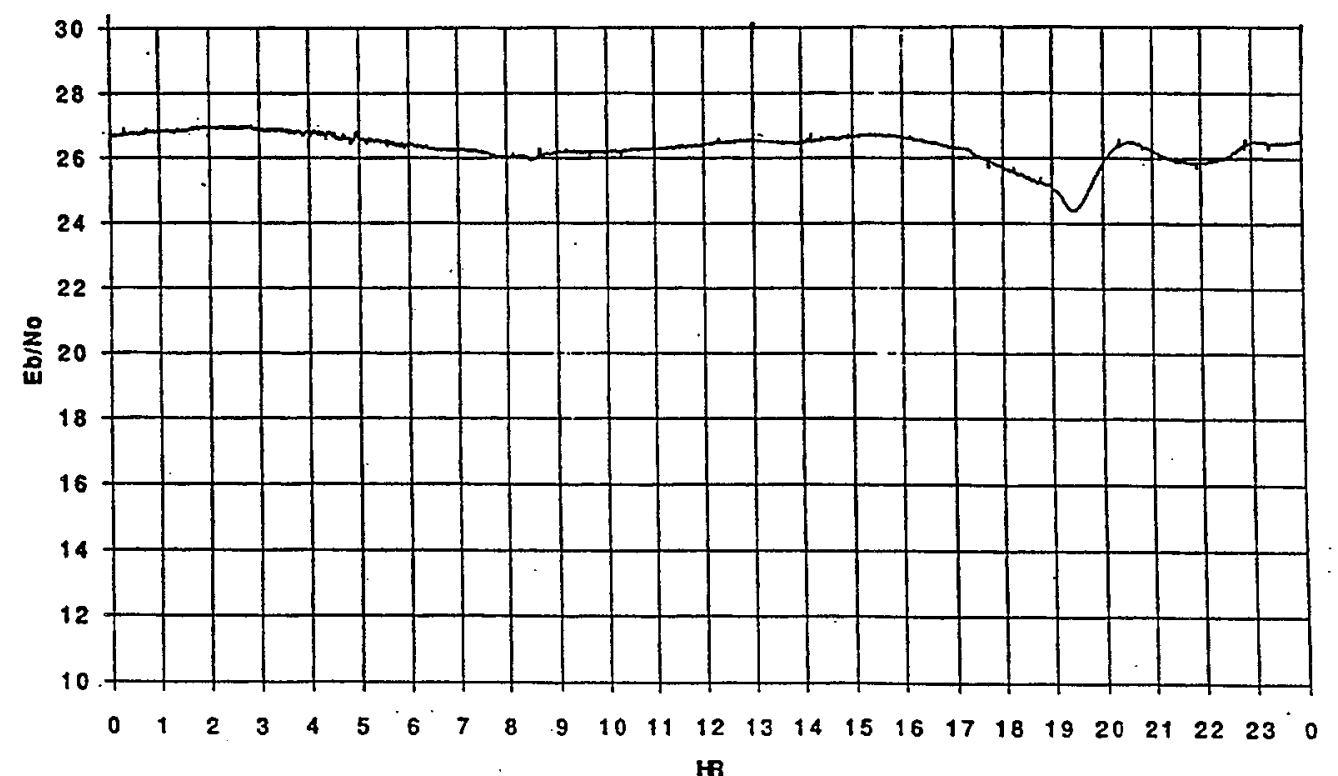

Figure 19 Typical downlink east beam received signal by a T1VSAT ground station as afunction of time of day. 


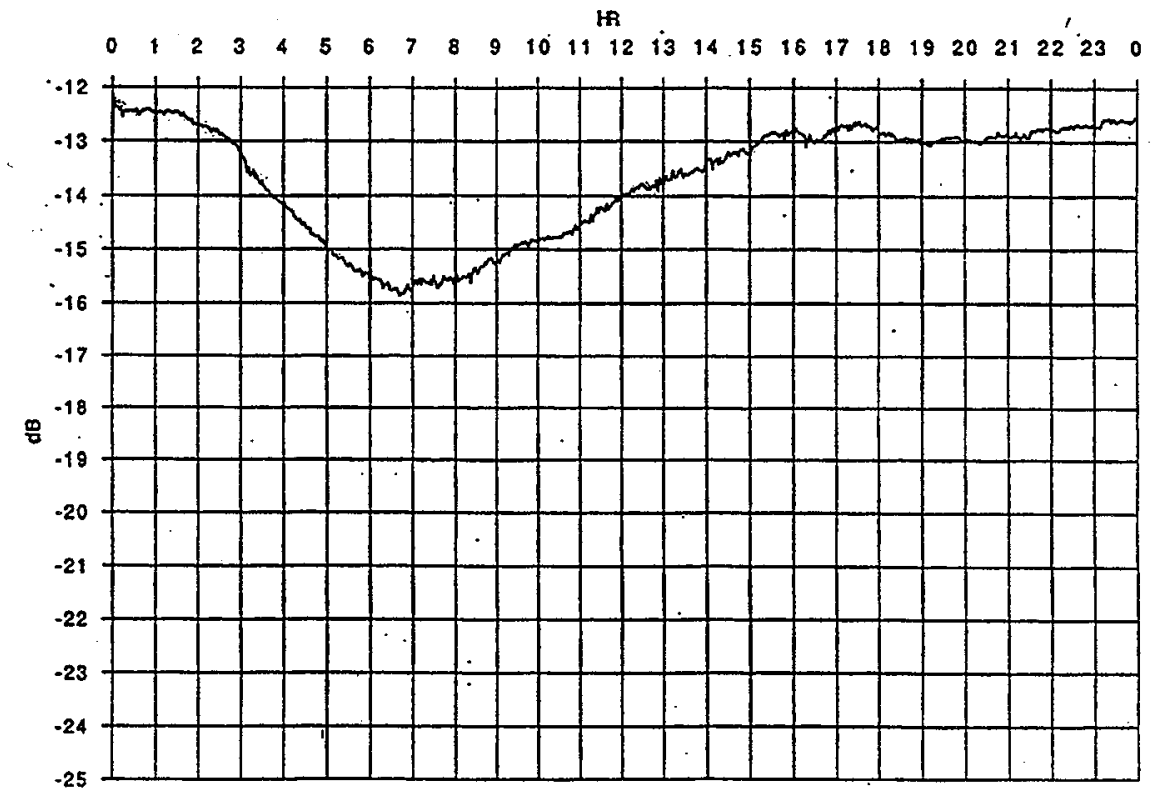

Figure 20 Typical downlink west beam received signal by the NASA (NGS) ground station as afunction of time of day.

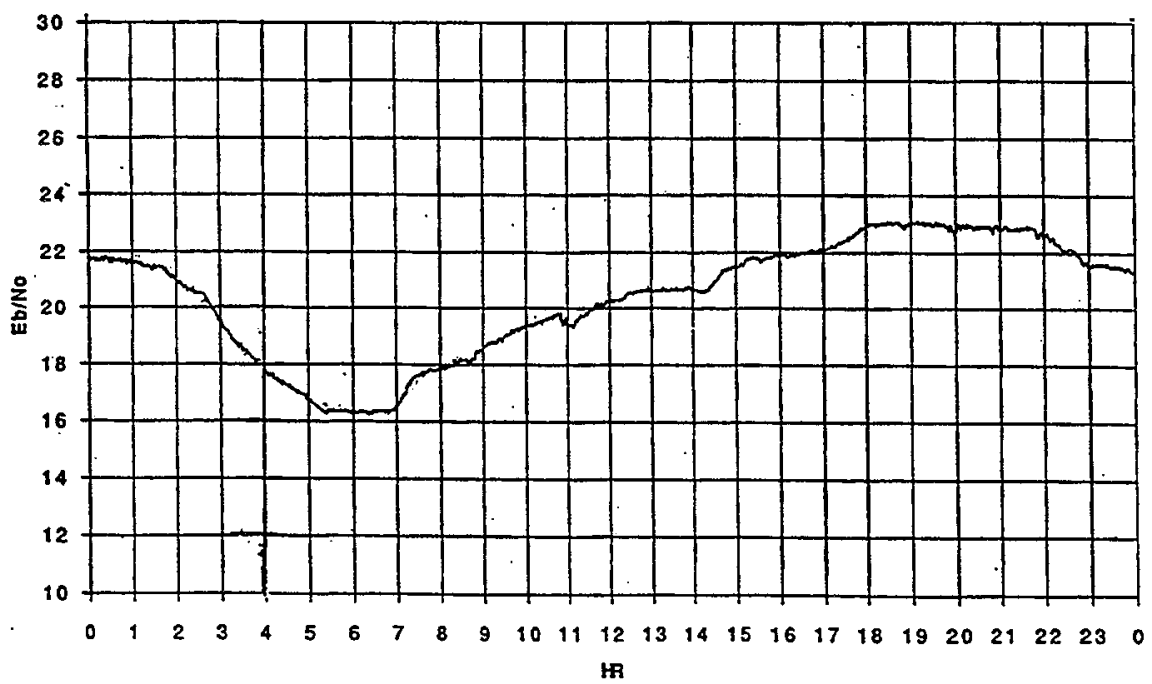

Figure 21 Typical downlink west beam received signal by the T1VSAT ground station as a function of time of day. 

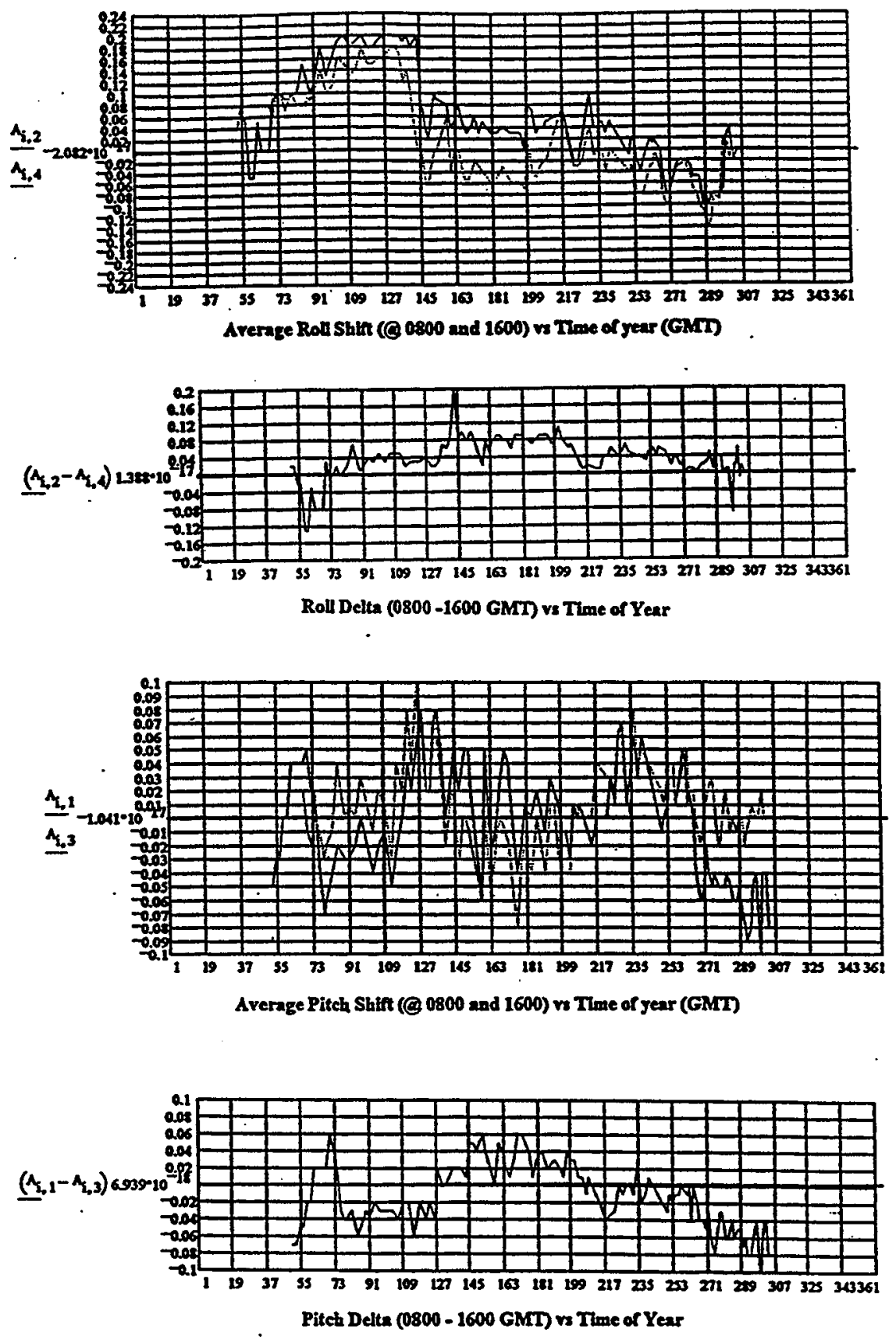

Figure 22 Quasistatic effect on transmit and receive beams. 


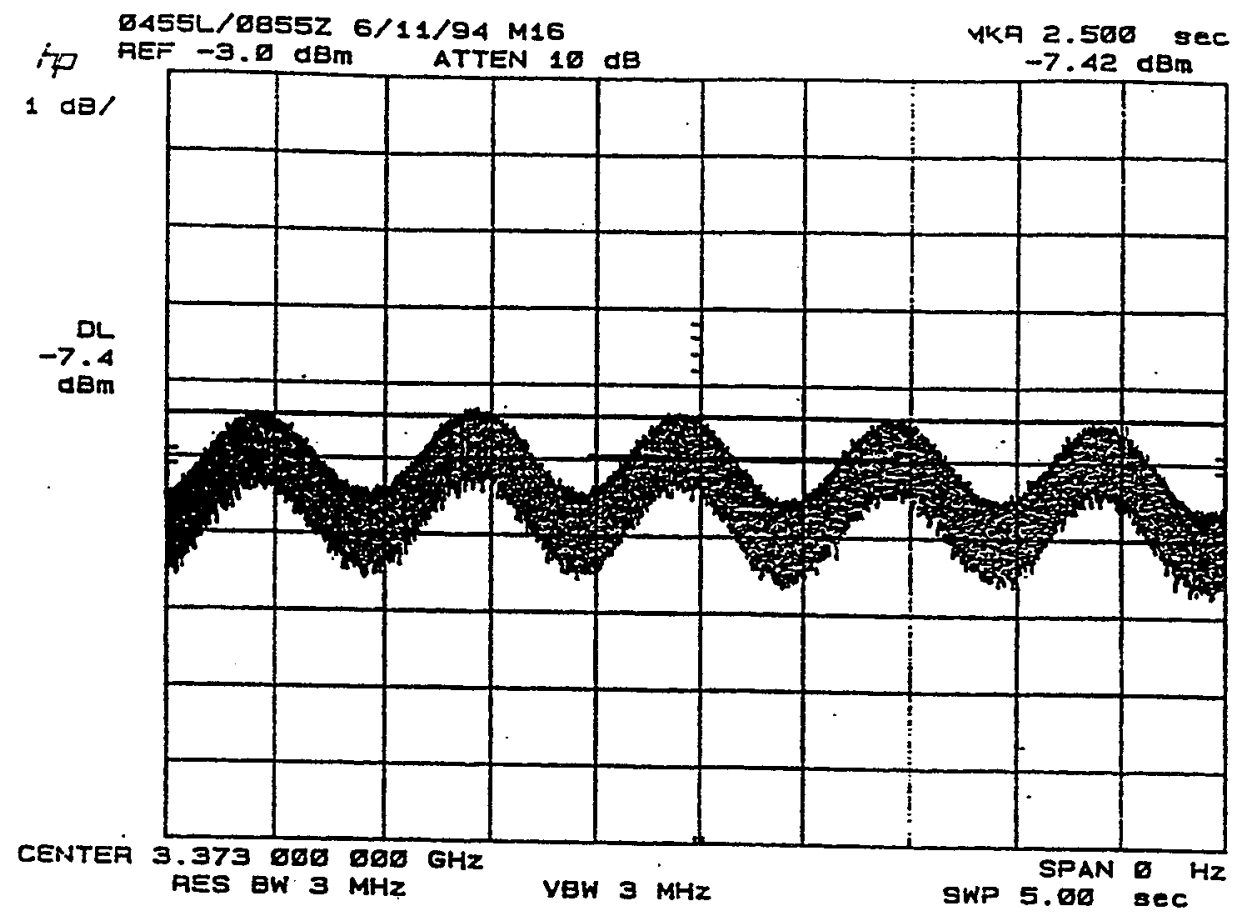

Figure 23 Received LET $20 \mathrm{Ghz}$ signal (Beam shifted 0.12 degrees).

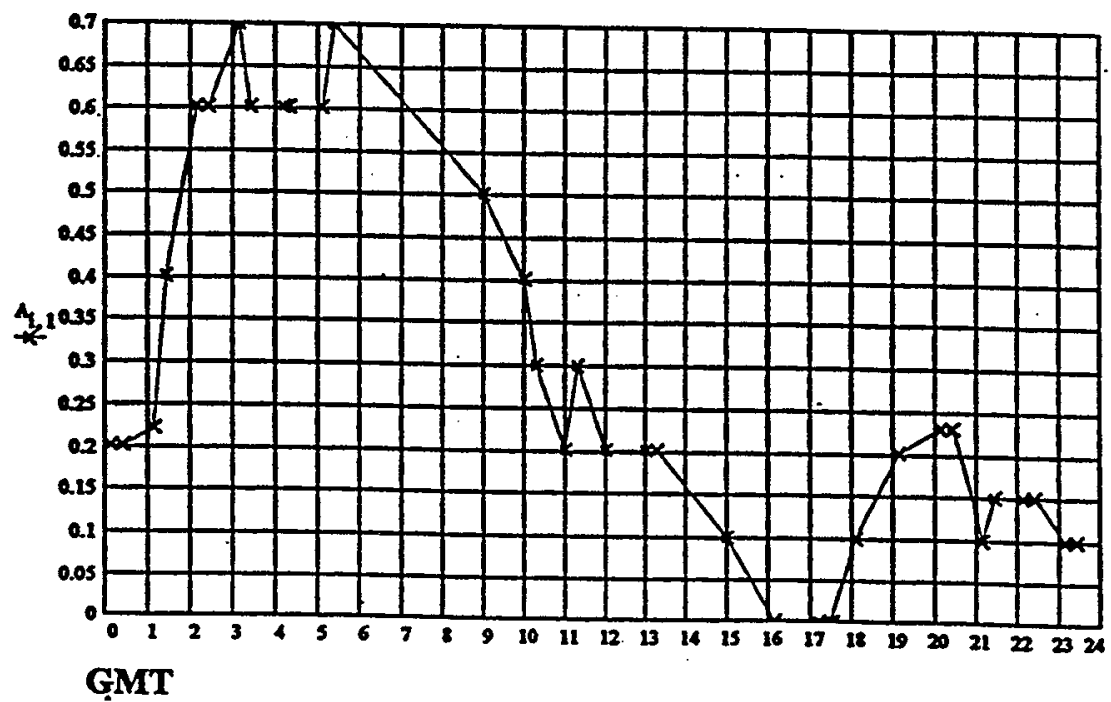

Figure 24 Amplitude distortion variation for a $24 \mathrm{hr}$. period. 


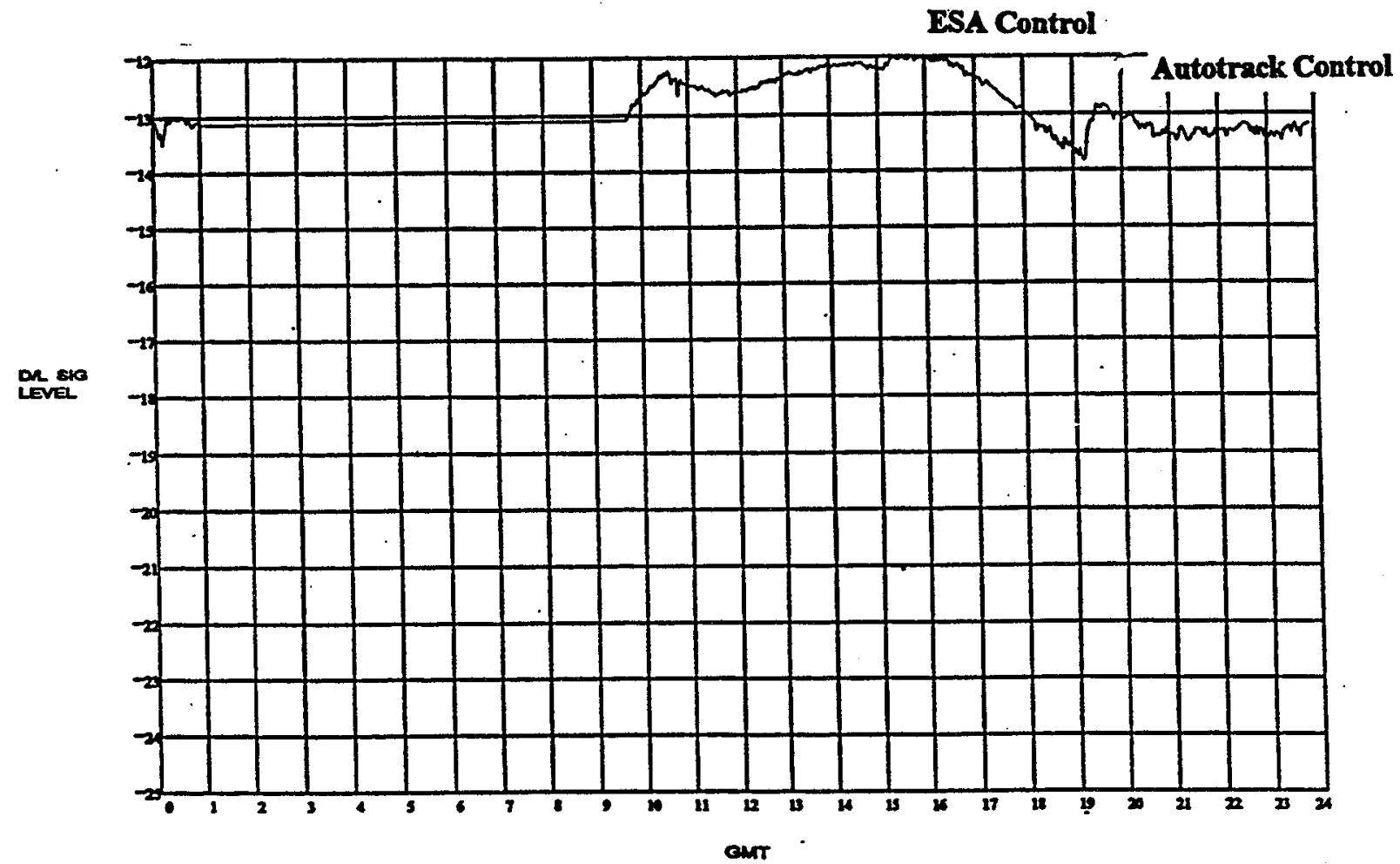

Figure 25 Combine yaw and roll error effects on the received signal at the NASA ground station (West Scan 08 beam) with the S/C on ESA and autotrack control. 

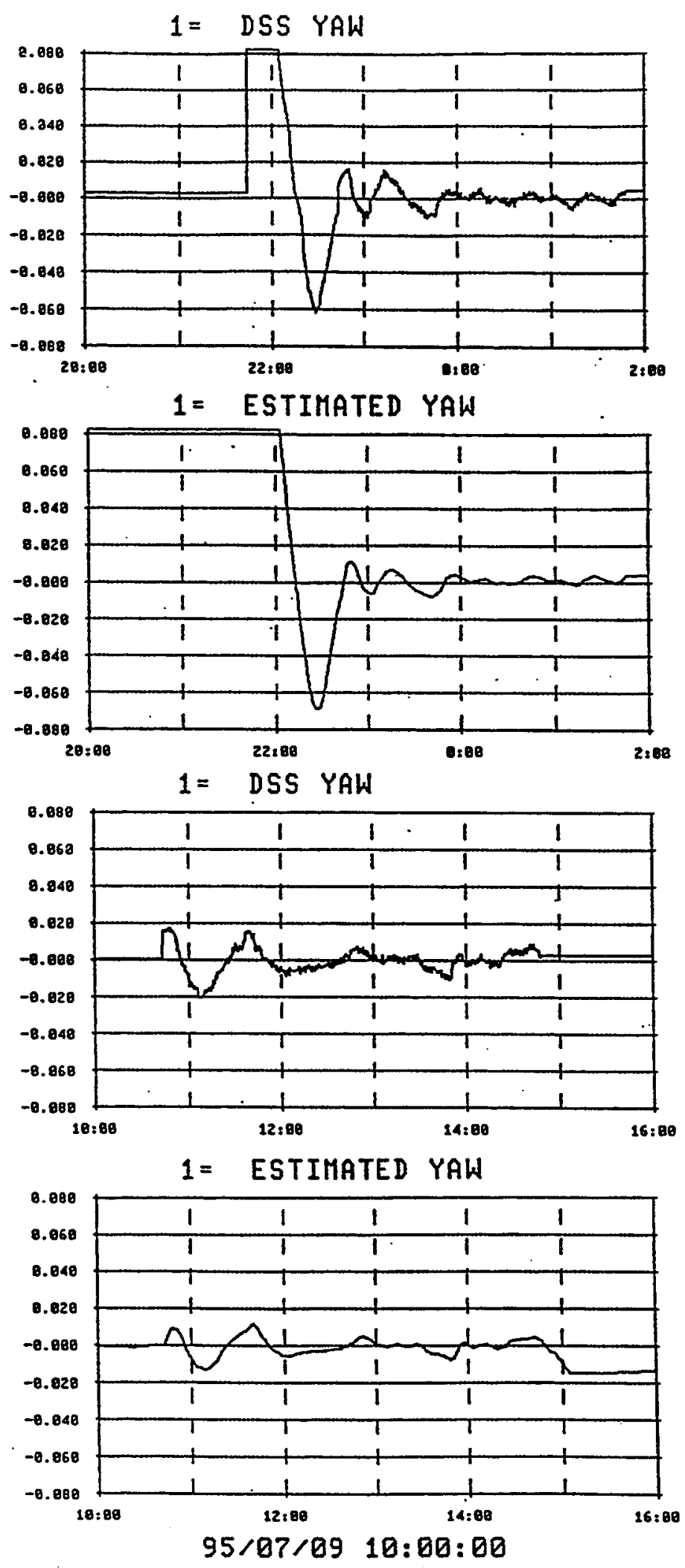

Figure 26 Estimated yaw error vs. actual yaw error during the east and west sun windows. 

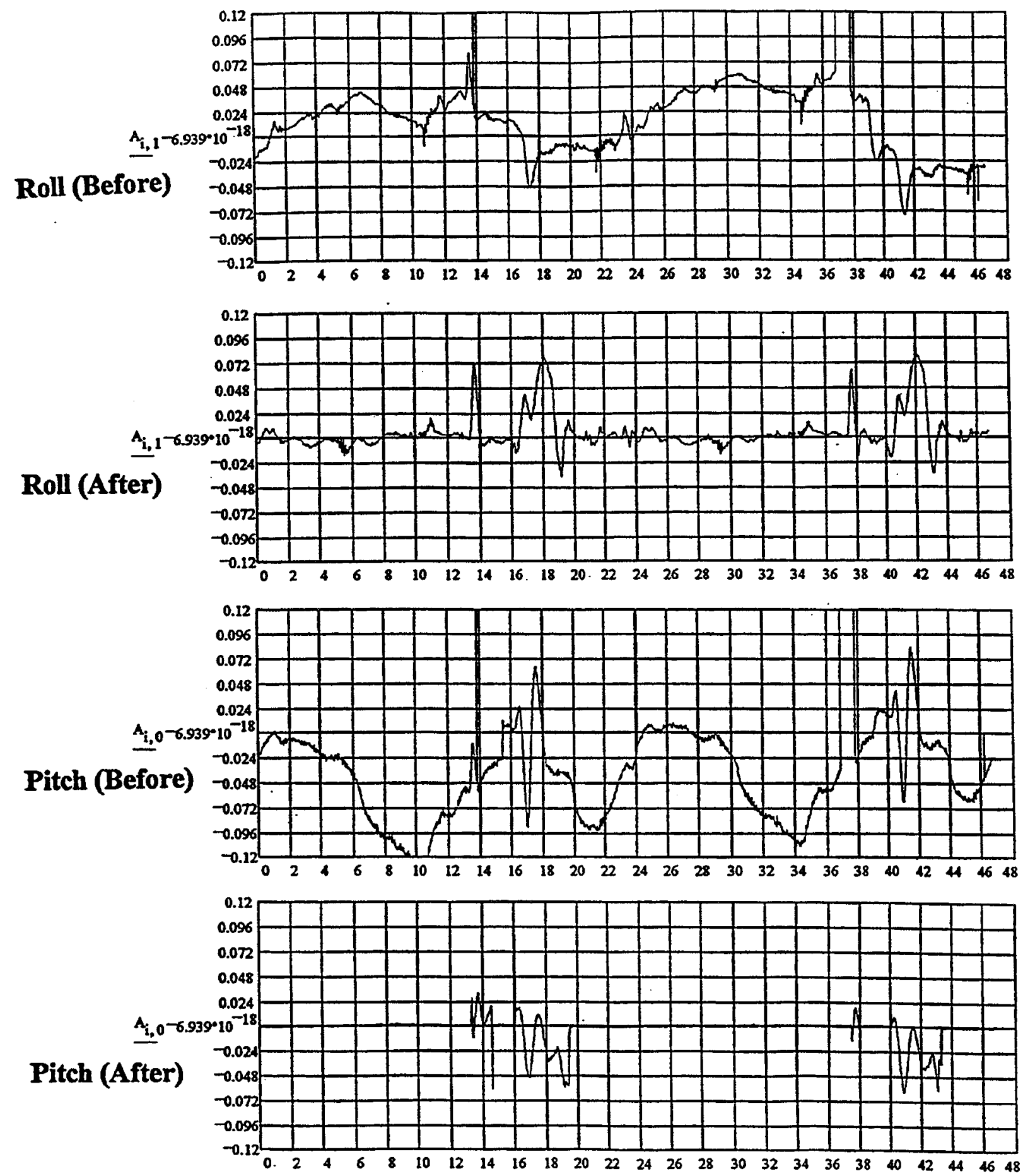

Figure 27 East receive autotrack error signal before and after compensation. 


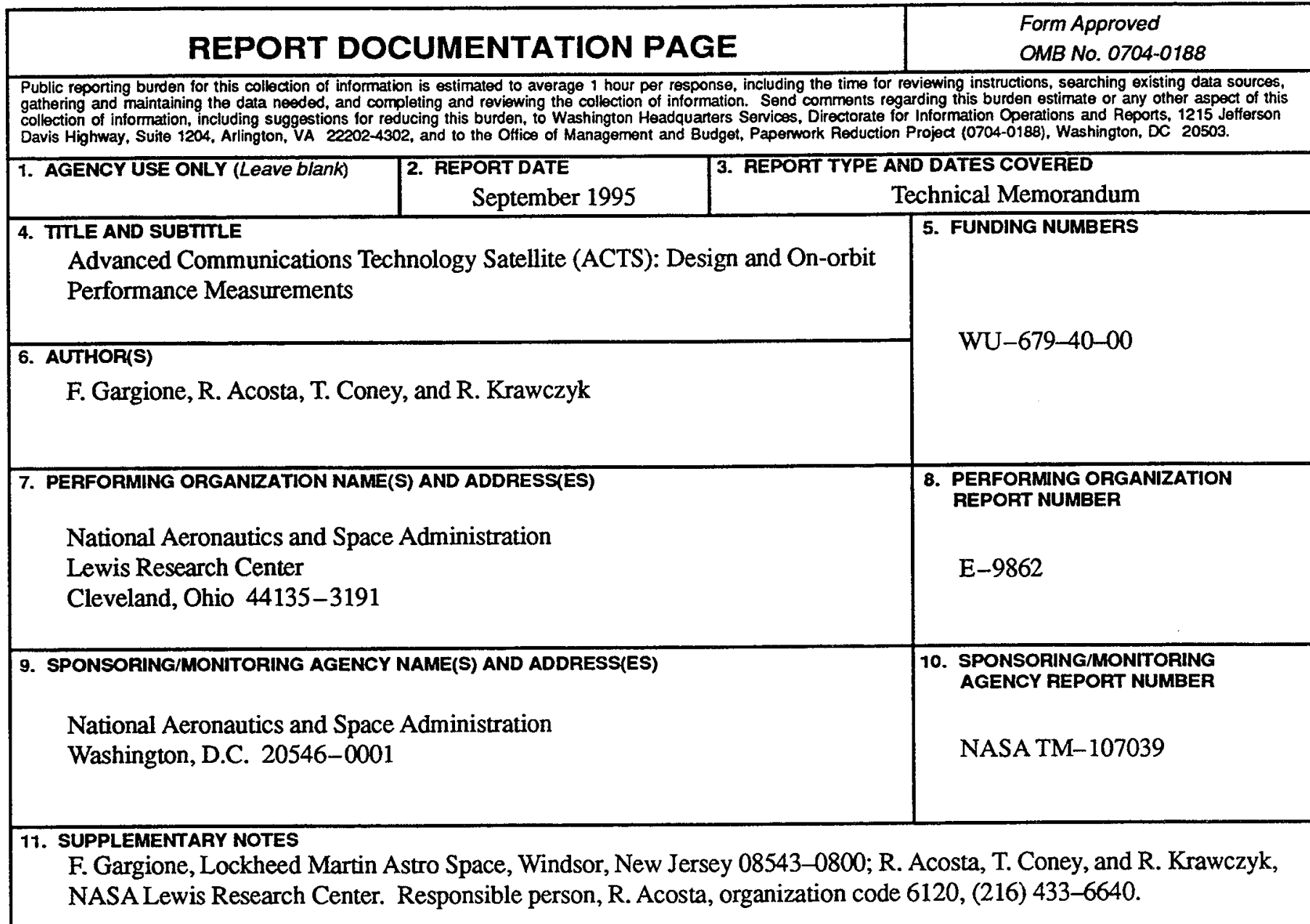

\begin{tabular}{|l|l|}
\hline 12a. DISTRIBUTIONAVAILABILTTY STATEMENT & 12b. DISTRIBUTION CODE \\
Unclassified - Unlimited & \\
Subject Category 32 & \\
This publication is available from the NASA Center for Aerospace Information, (301) 621-0390. & \\
\hline
\end{tabular}

13. ABSTRACT (Maximum 200 words)

The Advanced Communications Technology Satellite (ACTS), developed and built by Lockheed Martin Astro Space for the NASA Lewis Research Center, was launched in September 1993 on the Shuttle STS 51 mission. ACTS is a digital experimental communications test bed that incorporates gigahertz bandwidth transponders operating at Ka band, hopping spot beams, on-board storage and switching, and dynamic rain fade compensation. This paper describes the ACTS enabling technologies, the design of the communications payload, the constraints imposed on the spacecraft bus, and the measurements conducted to verify the performance of the system in orbit.

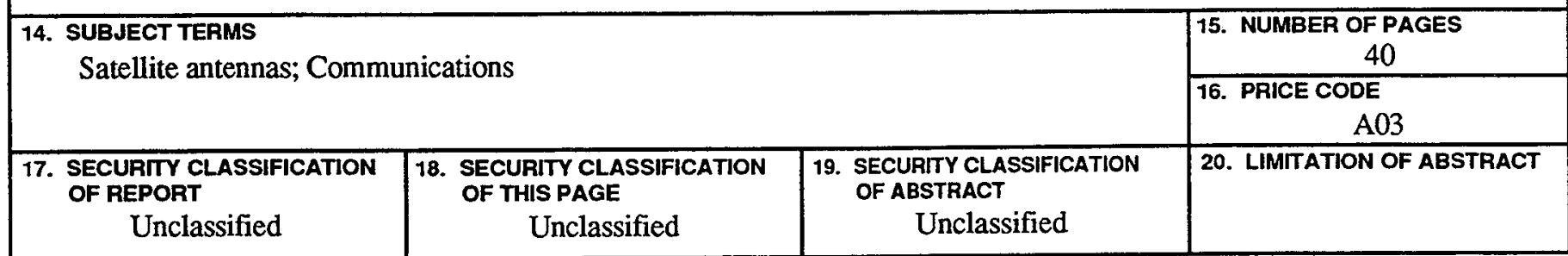

